\title{
MECHANICS AND PHYSIOLOGY OF ANIMAL SWIMMING
}

\author{
A Symposium organized by the Marine Biological Association and the Biomechanics \\ Group of the Society for Experimental Biology, and held at the Polytechnic South \\ West in Plymouth, 15-18 April 1991
}

Abstracts of papers and posters presented

\author{
Edited by J.M.V. Rayner \\ Department of Zoology, Woodland Road, University of Bristol, Bristol, BS8 1 UG
}

\section{PAPERS}

\section{Mechanical properties of the swimming and respiratory appendages of isopod crustaceans \\ David E. Alexander \& Jeff G. Blodig. Department of Entomology, Haworth Hall, University of Kansas, Lawrence, Kansas 66045-2106, USA}

Many isopod crustaceans use their abdominal appendages (pleopods) both as paddles for swimming propulsion and as gills. In Idotea wosnesenskii the anterior three of the five pairs of pleopods are used as paddles, but gas exchange occurs only in the posterior two pairs. We measured the mechanical stiffness of the pleopods and compared it within several individuals to relate the pleopods' stiffness to their function, as well as to evaluate constraints that might prevent gas exchange by the anterior pleopods. We bent pleopods with a calibrated micromanipulator, and measured the force for a given deflection by using an electronic balance; the slope of the bending force/deflection curve represented an index of flexural stiffness. The pleopods decreased in stiffness from anterior to posterior; furthermore the first and second pleopods were typically an order of magnitude stiffer than the fourth and fifth (respiratory) pleopods. Both internal blood flow through, and diffusion into the swimming pleopods may be limited by the thicker cuticle which probably causes the increased stiffness. Swimming requirements may thus preclude gas exchange by swimming pleopods.

\section{The mechanical properties of fish myotomal muscle during simulated swimming}

J.D. Altringham. Department of Pure and Applied Biology, The University, Leeds, LS2 9JT

During swimming, the locomotory muscles undergo cyclical length changes (strains), and are phasically activated in each tail-beat cycle. The amplitude and waveform of the strain cycle, and its relationship to muscle activity, have been determined for several species of fish during steadystate swimming. Patterns are characteristic of the species, swimming speed and location of the myotome. Guided by these studies, we have investigated the mechanics and energetics of isolated muscle fibre bundles under conditions which simulate their activity during swimming. The fibres are subjected to sinusoidal strains, whose amplitude and frequency ranges are those 
recorded in vivo. In each cycle, the fibres are activated by one or more supramaximal stimuli, and perform mechanical work. Net power output and other mechanical parameters are determined by the strain and stimulus parameters. The technique is being used to improve our understanding of how muscle is used during swimming, and to determine the mechanisms by which its properties are matched to the varied demands imposed on it. Aspects under study include: the role of different fibre types during swimming, the effects of body size on muscle function, the mechanochemical efficiency of myotomal muscle, and the activity patterns and power output of myotomes at different locations along the body.

\section{The swimming of spermatozoa with helical heads: theoretical analysis and experimental results}

\section{F. Andrietti \& G. Bernardini. Dipartimento di Biologia, Universita di Milano, via Celoria 26, I-20133 Milano, Italy}

Helical movements of flagellated micro-organisms with spherical heads have already been studied by many authors. The present note tries to extend the study to the case of helical heads, such as those shown by the spermatozoa of Xenopus. The theoretical approach is similar to that presented by Chang \& Wu for the study of helical flagellar movement, and is done according to the theory of Gray \& Hancock.

The biomechanical model used in the present study allows determination of the velocity of propulsion $(U)$ and the frequency of rotation of the head $\left(f_{h}\right)$ as a function of the frequency of the wave of motion travelling along the tail $\left(f_{t}\right)$. The results relative to the case of helical and planar flagellar waves are compared. The main result to stress is that the helical shape of the head seems to increase considerably the velocity of propulsion of the spermatozoon compared to that of the more common spherical head. Experimental measured values of $f_{h}$ versus $U$ may be fitted by a linear plot that lies midway between that relative to the helical and that relative to the planar flagellar wave. This suggests the possibility that the shape of the flagellar movement conforms to a flattened helix with an elliptical cross section.

\section{Functional anatomy of the 'flight' apparatus in penguins}

R. Bannasch. Forschungsstelle für Wirbeltierforschung, Alfred-Kowalke-Straße 17, D-1136 Berlin, Germany

Penguins are the best-adapted wing-propelled divers in birds. It seems that their complete change from aerial to underwater flight is realized without any compromises.

In water the weight of their nearly perfectly streamlined body is balanced by the buoyancy, hence the strong 'flight' apparatus is used only for thrust production.

Similar to flying birds penguins flap their wings. But, contrary to the fast flight in air, thrust is produced under water also during the upstroke. Corresponding wing profiles are developed and the articulations inside of the wing are bared. Since the wings are permanently active in an extended position the shoulder joint is simplified. The examination of its structure and kinematics reveals a new type of articulation, never previously described. The 'rotation' of the head of the humerus in the shoulder joint can be described as a two-axis-eccentric motion conducted by the ligamentum acrocoracohumerale and an articulation surface of the Caput humeri shaped like a logarithmic spiral. This model leads to new interpretations of the function of the muscle system.

Besides the thrust the flapping wings produce also positive and negative 'lifting' forces during the downstroke and upstroke, respectively. In non-accelerated forward swimming the impulses perpendicular to the direction of translational motion must be balanced. Therefore the simplest solution would be mirror symmetry of up- and down-stroke. But several details in the construc- 
tion of the locomotor system do not fully correspond to this idea. Thus, for example, power transmission during the upstroke is achieved by secondary adaptations which are difficult to access in view of their efficiency. Generally the penguins have to live with several compromises still persisting from their flying ancestors and obstructing much simpler constructions.

Also the few kinematic pictures available from underwater flight of penguins show the symmetry of up- and down-stroke to be only optional. This opens a broad field for interpretation. But more detailed kinematic studies combined with metabolic measurements are required to connect the anatomical, kinematic and energetic data in a single model of the underwater flight of penguins.

\section{Swimming performance in anostracan and copepod nauplii}

\section{Barlow \& M.A. Sleigh. Department of Biology, School of Biological Sciences, University of} Southampton, Bassett Crescent East, Southampton, SO9 $3 T U$

The problems of swimming at intermediate Reynolds numbers call for compromise strategies by those organisms that are subjected to them.

Different strategies are exhibited by anostracan and copepod nauplii. Artemia saline nauplii use a single pair of rowing limbs, a strategy also found in the diving beetles. On the other hand, the nauplii of Eucyclops agilis and Cyclops strenuus abyssorum show multi-limb propulsion.

These nauplii provide model systems for the comparison of the efficiency of rowing propulsion at intermediate Reynolds numbers $(1-10)$ by single and multiple pairs of rowing limbs. The Artemia nauplii and meta-nauplii show poor swimming performance when compared to the copepods. However, all the nauplii show a much more sophisticated control of swimming activity than had been thought previously.

By measuring the power in the output flow from the limbs of tethered specimens, it was possible to obtain some estimates of the mechanical power output of the nauplii during active swimming for comparison with previous estimates obtained for diving beetles and adult copepods. These results might suggest that swimming strategy in nauplii may well be dictated more by ecological pressures than by physical ones.

\section{The swimming of fish larvae studied by high-speed video}

\section{R.S. Batty \& J.H.S. Blaxter. Dunstaffnage Marine Laboratory, PO Box 3, Oban, Argyll, PA34 4AD}

A NAC-corp high-speed video operating at 200 or 400 frames $\mathrm{s}^{-1}$, with associated MOVIAS software, was used to study the fast-startle responses (C-starts) of herring, plaice, flounder and sole larvae soon after hatching. The larvae were induced to swim by administering tactile stimuli with a probe. The following parameters were measured: latency of the response, C-start contraction time, yaw, tail beat frequency and amplitude, acceleration, swimming speed, stride length and number of body waves. The effect of temperature was also studied. The results are related to hydrodynamic considerations such as Reynolds number.

\section{New aspects of the energetics of fish locomotion}

\section{R. Blickhan \& W. Nachtigall. Fachrichtung Zoologie, Unizersität des Saarlandes,} D-6600 Saarbrïcken, Germany

So far the mechanical energy necessary to sustain steady swimming has been estimated either from drag or from hydrodynamic models. Both methods are prone to errors owing to their respective assumptions. In order to obtain an independent measure for the mechanical cost of locomotion, we investigated the flow field in the wake of a freely swimming rainbow trout. Drag 
during undulatory locomotion as estimated from the vortices in the wake is similar to values measured on gliding animals ( $c w s=0.007$ to 0.011 , at $R e=6.4 \times 10^{5}$ ). Separation and thus an increase of pressure drag is avoided by the undulatory pump.

It becomes more and more evident that the axial musculature does not simply operate at the point of maximum power output but is partially prestretched. Nevertheless, the question of energy storage in elastic tissues remains unresolved. We investigated the mechanical properties of fish skin by dynamic tensile tests and measured strain in the skin during locomotion. The energies necessary to stretch the skin and to overcome drag are of similar magnitude. About $70 \%$ of this energy can be recovered.

\section{Streamlined body shape in amphipods (Crustacea: Peracaridea) swimming at intermediate Reynolds number}

\section{M.A. Boudrias. Scripps Institution of Oceanography, University of California at San Diego, La Jolla, California 92093, USA}

Amphipod crustaceans with pelagic and benthic lifestyles range in length from a few $\mathrm{mm}$ to more than $25 \mathrm{~cm}$, yet most species exhibit a streamlined body shape while swimming. In particular, the deep-sea necrophagous lysianassids have body diameter to length ratios akin to high-performance swimmers such as tuna and dolphins. Their ambit covers several $100 \mathrm{~m}$ horizontally and more than $1 \mathrm{~km}$ vertically. Their Reynolds numbers, as well as that of many other swimming crustaceans, range from 100 to less than $10^{5}$. Theoretical and empirical determinations of drag coefficients $\left(C_{d}\right)$ for standard engineering airfoils at Reynolds number $<10^{5}$ suggest that streamlining is no longer important. The values of $C_{\mathrm{d}}$ for various airfoils are between 0.05 and $0 \cdot 2$. Using theory developed to calculate $C_{d}$ for streamlined bodies of revolution, I found that drag coefficients for several amphipod species were an order of magnitude less than predicted by standard airfoil theory. To verify my theoretical calculations, empirical drag measurements were done in a newly designed tow tank. The empirical drag coefficients range from 0.006 to 0.078 , agreeing well with calculations based on streamlined bodies of revolution. These results suggest that streamlining may confer drag reducing advantages for amphipods even at intermediate Reynolds number.

\section{A mathematical model of lamprey body mechanics}

\section{Graham Bowtell. Mathematics Department, City University, Northampton Square, London, EC1V OHB}

\section{Thelma L. Williams. Department of Physiology, St George's Hospital Medical School, University of London, Cranmer Terrace, Tooting, London, SW17 ORE}

A simple two-dimensional rod and pivot model is proposed for the mechanical structure of the lamprey, each pivot being controlled by a muscle segment attached via perpendicular extensions to the two rods. The elastic and viscous properties of the body tissues (including muscle) are described as linear functions of the relative displacement and angular velocity of the rods at each pivot. The contractile properties of the muscle are introduced as time-dependent forcing torques at the pivots, which in turn are generated by a travelling wave of activation. The linearized equations of motion for the adapted coordinates of angles between the rods at each pivot correspond to low curvature dynamics. Investigation of these equations with varying viscous and elastic parameters leads to a reconstruction of a lamprey viewed in motion on a smooth flat surface out of water. The most striking feature is of an apparently standing wave motion, which is indeed observed in the real animal but which on careful examination in the model corresponds to a travelling wave of varying amplitude. 


\section{Lamprey hydrodynamics: the numerical solution of the Navier-Stokes equations}

John Carling \& Thelma L. Williams. Department of Physiology, St George's Hospital Medical School, University of London, Tooting, London, SW17 ORE

The solution of anguilliform hydrodynamics is required to understand how the observed patterns of muscle activation in the lamprey give rise to swimming. The approach used in the present work is to solve numerically the momentum equations and the continuity equation governing the hydrodynamics. To facilitate this solution, a coordinate transformation has been used which captures the shape of the lamprey as it moves through the water. This paper first describes the coordinate transformation approach in general terms, together with a summary of the longer-term aims of the research. Some preliminary work is then described involving the computation of the flow produced by the movement of a hypothetical 'square-wave' lamprey. Results are presented showing how pressure distribution, velocity vectors and drag change with time as this hypothetical creature moves along its path. Finally these results are discussed in relation to the swimming behaviour of a realistic anguilliform creature.

\section{Invertebrate swimming: the dilemma of matching muscle mechanics to fluid forces}

\section{T.L. Daniel. Department of Zoology, NJ-15, University of Washington, Seattle, Washington 98195, USA}

Models and measurements for swimming animals suggest that there are distinct relationships between the speed of appendage or body motions and the hydrodynamic forces such structures produce. Physiological and biomechanical studies of muscle contraction, however, suggest there are quite different relationships between force generation and appendage speeds. Both relationships must be satisfied for the prescribed movements to be physically and physiologically feasible. I use simple hydrodynamic models of escape swimming in shrimp and rowing motions of aquatic insects to show how both appendage movements and body trajectories may be predicted from morphological and physiological characteristics. Moreover, I argue that the use of hydrodynamic models alone to suggest optimal motions and morphologies may lead to physiological dilemmas.

\section{Fuel homeostasis in the harbour seal during submerged swimming}

\section{Randall W. Davis. Department of Marine Biology, Texas AEM University, PO Box 1675, Galveston,} Texas 77573, USA

The turnover rates and oxidation rates of plasma glucose, lactate, free fatty acids and triglycerides were measured in three harbour seals at rest or during voluntary submerged swimming in a water flume at $35 \%\left(1.3 \mathrm{~m} \mathrm{~s}^{-1}\right)$ and $50 \%\left(2 \mathrm{~m} \mathrm{~s}^{-1}\right)$ of maximum oxygen consumption. At $35 \%$ and $50 \%$ maximum oxygen consumption, glucose turnover and oxidation rates did not change from resting levels. Glucose oxidation contributed about $1 \%$ of the total ATP production during swimming. The plasma free fatty acid oxidation rate at $50 \%$ maximum oxygen consumption increased almost 3.5-fold and accounted for $21 \%$ of ATP production. The maximum oxidation rate of triglycerides contributed $31 \%$ of ATP production at rest. Dive duration decreased from $78 \mathrm{~s}$ while resting in water to $28 \mathrm{~s}$ at $50 \%$ maximum oxygen consumption. The RQ ranged from 0.78 at rest to 0.74 at $50 \%$ maximum oxygen consumption, indicating that fat was the primary source of energy during submerged swimming. Anaerobic metabolism contributed about $1 \%$ of the total ATP production at rest and during swimming. By adjusting breath-hold duration during strenuous underwater swimming, harbour seals are able to maintain an aerobic, fat-based metabolism. 


\section{Diversity of swimming in the invertebrates \\ M. Edwin DeMont. Department of Biology, St Francis Xavier University, Antigonish, Nova Scotia B2G 1CO, Canada}

This paper surveys the swimming modes and hydrodynamics of swimming invertebrates. Soft bodied animals use a vast collection of mechanisms to swim. The best swimmers use jet propulsion, although this is inherently inefficient. Fin based swimming has appeared in the cephalopods, and may be a mechanism to overcome the inherent inefficiency. Elastic mechanisms in the body wall of several swimmers have increased the efficiency of refilling. I review jet-propelled swimming in a diversity of animals, including squid, scallops, Nautilus, jellyfish and tunicates. Some invertebrates, mostly those that have a rigid exoskeleton, use limbs to propel themselves with a rowing action. The biomechanics and hydrodynamics of this form of locomotion are discussed.

\section{Shark and tuna swimming kinematics and fin-fin interactions}

H. Dewar \& J.B. Graham. Physiological Research Laboratory, Scripps Institution of Oceanography, University of California at San Diego, La Jolla, California 92093, USA

Kinematic analyses of swimming in three tuna and two shark species is in progress using a large water tunnel, video equipment and dye streams. Regressions of tail-beat frequency (TBF) and stride length $(S L)$ on velocity indicate that $S \mathrm{~L}$ increases with size and velocity in tuna. At a swimming velocity of $1.8 \mathrm{~L} \mathrm{~s}^{-1}$, Thunnus albacares $(50 \mathrm{~cm}$ fork length, $L)$ has a greater $S L(0 \cdot 6 \mathrm{~L})$ than skipjack of equal size $(0.47 \mathrm{~L})$. Observations in tunas of burst-glide behaviour and decreased TBF at high temperatures $\left(26-30^{\circ} \mathrm{C}\right.$ over a range of $\left.18-30^{\circ} \mathrm{C}\right)$ indicate modulation of kinematic parameters with temperature which may result from thermoregulatory mechanisms or changes in dynamic viscosity and muscle properties. Dye streams emitted from the first dorsal (Triakis semifasciata) and second dorsal (Thunnus albacares) fins show a phase difference between the vortex sheet shed by the anterior fin and the caudal fin itself. Fin-fin interactions in sharks may allow a greater contribution of the anterior fin to thrust. In tunas, for which no such fin-fin interaction has been proposed, the rotation of the second dorsal and anal fins may facilitate the phase difference, reducing the turbulence encountered by the caudal fin, increasing thrust.

\section{The escape response in the angelfish (Pterophyllum eimekei)}

Paolo Domenici \& Robert W. Blake. Department of Zoology, University of British Columbia, 6270 University Boulevard, Vancouver, British Columbia V6T 2A9, Canada

Although angelfish (Pterophyllum eimekei) swim routinely employing pectoral fin (labriform) locomotion at low speed, they are capable of escape responses employing axial movements. The turning kinematics and performance of the response is analyzed and two kinds of C-starts are observed: a single bend start, in which the fish continues the turn initiated by the first body bend (stage 1), and a double bend start, in which a contralateral bend (stage 2) follows the first contraction (stage 1). The two responses have similar stage 1 kinematics, but differ in escape trajectory and performance. Higher distance-time performances associated with smaller angles of turn are found in double bend than in single bend responses. Escape trajectory and stage 2 turning angle can be predicted by stage 1 turning angle, which in turn is related to stage 1 duration with a size effect. Despite specialization for low-speed swimming, angelfish have good fast-start capability when compared to other teleosts. 


\section{Swimming in protists}

J. Febvre \& Colette Febvre-Chevalier. Université de Nice, F-06034 Nice, France \& Laboratoire de Biologie Cellulaire Marine, URA 671 CNRS, Observatoire Océanologique de Villefranche-sur-Mer, F-06230 Villefranche-sur-Mer, France

Motile behaviour, kinetics and cellular mechanisms involved in floating and swimming are reviewed in diverse marine pelagic protists. Attention is focussed on some unconventional systems of microtubule and/or nonactin filament-based motility that are directly or indirectly implicated in both of these functions, and a video-film is presented.

Examples of buoyancy regulation are taken among Foraminifera, Radiolaria and Acantharia. Reaction propulsion, rowing swimming, flying-like motion, top-like forward swimming and reorientation of the propagation direction are examined in various species of Acantharia, Heliozoa, Dinoflagellates and Ciliates.

\section{Pacific mackerel (Scomber japonicus) show increased locomotory efficiency when swum in groups}

\section{P.A. Fields. Physiological Research Laboratory, Scripps Institution of Oceanography, University of California at San Diego, La Jolla, California 92093, USA}

By using a 2800-l recirculating water tunnel, swimming effort was determined for eight Pacific mackerel, through use of tail-beat frequency $(T B F)$. The mackerel were swum alone and in groups of three at four speeds $\left(32,42,56\right.$, and $\left.69 \mathrm{~cm} \mathrm{~s}^{-1}\right)$. Regression analyses of $T B F$-speed showed that five of the eight fish had significantly lower $T B F$ when swimming in groups than they did when swimming alone. Further, in four of the eight fish, $T B F$ values in groups increased more slowly with respect to speed than they did when swimming alone.

Video analysis of dye streams is currently in progress to examine the positions of vortices shed by the caudal fin of swimming mackerel. These vortex positions are compared to the positions mackerel choose when swimming in groups. This tests the hypothesis that mackerel are able to use the hydrodynamic energy of vortices shed by others to enhance their own swimming efficiency.

\section{Energy conservation by formation swimming in ducklings}

F.E. Fish. Department of Biology, West Chester University, West Chester, Pennsylvania 19380, USA

Formation movement by swimming mallard ducklings (Anas platyrhynchos) was tested as a mechanism for energy reduction. Newly-hatched ducklings were imprinted to an adult decoy and trained to follow the decoy in a water current channel with a constant velocity of $0.3 \mathrm{~m} \mathrm{~s}^{-1}$. Clutches of one, two and four ducklings at ages of three, seven, and fourteen days were tested with the decoy in water or suspended above water. The entire clutch and decoy were enclosed in a metabolic chamber to monitor oxygen consumption. Ducklings swam in the decoy's wake in formations organized in single-file lines or echelons. Inter-duckling distances were less than one body length. The metabolic rate per individual for all ages decreased with increasing clutch size by $28 \cdot 1-56 \cdot 1 \%$. The influence of the wake of the decoy significantly decreased metabolic swimming effort by the ducklings at three days of age and in small clutches. I propose that the flow pattern generated by ducklings swimming in formation and in the wake of an adult reduces the resistance of the water to locomotion and allows for the conservation of energy on an individual basis. 


\section{Water tunnel studies with pelagic fishes}

J.B. Graham, H. Dewar \& N.C. Lai. Physiological Research Laboratory, Scripps Institution of Oceanography, University of California at San Diego, La Jolla, California 92093, USA

A 3000-1 portable water tunnel enabled laboratory and shipboard studies of shark (Triakis, Negaprion, Isurus) swimming performance, including cardiovascular function and blood-respiratory properties, tail-beat frequency $(T B F)$ in relation to velocity $(U)$, critical $U$, the $U$ of transition from cyclic to ram ventilation, and both EMGs and $\mathrm{O}_{2}$ uptake $\left(\mathrm{VO}_{2}\right)$ in relation to $U$. Findings to date are that shark swimming $V \mathrm{O}_{2}$ approaches that of cruise-adapted teleosts but that the range of $U$ in sharks is less. Use of this unit in Hawaii enabled the first-ever studies of tuna (yellowfin, Thunnus albacares; skipjack, Katsuwonus pelamis; kawakawa, Euthynnus affinis) swimming energetics and kinematics at controlled velocity and temperature and also allows close examination of yaw and yaw-eye compensation, pectoral fin sweep angle in relation to $U$, and finlet action. Thermal, scaling, and $\mathrm{U}$ effects on $\mathrm{VO}_{2}$ have been documented as has the shift, at warmer temperatures, from a steady $T B F$ to a burst-glide mode in yellowfin and skipjack. This may be related to heat balance and thermal changes in muscle efficiency and water viscosity.

\section{Thrust production and power requirements of a flexible wing at high amplitude}

Daniel Grunbaum. Section of Ecology and Systematics, Cornell University, Ithaca, New York, USA

Rapid undulation of thin, high aspect-ratio wings and fins is a common mode of thrust and lift generation among a diverse range of aquatic animals. The unsteady forces resulting from this motion are described by the Euler equations for the motion of an inviscid, incompressible fluid. Attempts to predict forces and energy requirements for such motions have relied on analytical solutions to the linearized Euler equations. The linearized equations concern the assumption that the amplitude of undulations is small relative to chord length. However, for many creatures, appendage motions are so large as to violate this assumption. In this study, an efficient numerical scheme is used to solve the full Euler equations for biologically realistic amplitude ranges. Preliminary results indicate that significant deviations from linear theory occur at amplitude to chord ratios larger than approximately $0 \cdot 1$. Power requirements increase faster with increasing amplitude than predicted by linear theory. Thrust production increases are more nearly consistent with linear predictions. The result is an overall loss of efficiency with increasing amplitude.

\section{Muscle contraction time and swimming speeds of the Atlantic cod at low temperatures}

P. He. Newfoundland and Labrador Institute of Fisheries and Marine Technology, PO Box 4920, 155 Ridge Road, St John's, Newfoundland A1C 5R3, Canada

C.S. Wardle. DAFS Marine Laboratory, PO Box 101, Victoria Road, Torry, Aberdeen, AB9 8DB

Twitch contraction time of the white muscle of the Atlantic cod, Gadus morhua L., was measured at the acclimated temperatures of $0^{\circ} \mathrm{C}$ in Newfoundland and $7^{\circ} \mathrm{C}$ in the North Sea. The shortest contraction times at the head end of the $0^{\circ} \mathrm{C}$ cod are between 60 and $91 \mathrm{~ms}$, while those of the $7^{\circ} \mathrm{C}$ cod are between 27 and $37 \mathrm{~ms}$. When the muscle of the $0^{\circ} \mathrm{C}$ cod was warmed up to $7^{\circ} \mathrm{C}$, the contraction time is similar to that of $7^{\circ} \mathrm{C} \operatorname{cod}$ measured at the same temperature. This indicates that adaptation of muscle contraction properties did not occur over this temperature range. Predictions of the maximum swimming speeds from the measured contraction time indicate that cod in the North Sea may be able to swim twice as fast as the same species of fish in Newfoundland waters at their typical winter temperatures of $7^{\circ} \mathrm{C}$ and $0^{\circ} \mathrm{C}$ respectively. Endurance and swimming speed of cod were measured at around $0^{\circ} \mathrm{C}$ in Newfoundland. A significant 
reduction in both swimming speed and endurance was recorded at these low temperatures when compared with those measured at higher temperatures. Reductions in both maximum and sustained ability of fish due to low temperature has a significant implication in their survival in terms of escape from a predator and fishing gear, and capture of prey.

\section{Mechanics of flapping ray locomotion \\ C. Heine. Department of Zoology, Duke University, Durham, North Carolina 27706, USA}

Two families of rays, the Myliobatididae and the Mobulidae, swim by using large amplitude flapping motions of their pectoral fins. Two species of the former family, the cownose ray Rhinoptera bonasus and the bullnose ray Myliobatis freminvilei, were filmed swimming in a recirculating flow tank at the Duke University Marine Laboratory. The animals' movements were quantified and a mathematical description of the motion was developed. Lift and drag coefficients for a series of fin elements were measured by using casts of rays in a wind tunnel. The kinematics of these rays are unique in that their fins oscillate, at approximately $1 \mathrm{~Hz}$, perpendicular to the direction of the animals' propagation twisting less than 25 degrees from that direction. The advance ratio $(J=V / n d)$, calculated by using the maximum tip speed, is unusually high with a minimum value of 4 , indicating that rays are not using a typical propeller-like mechanism for generating thrust. A hypothesis for the mechanics of thrust generation has been developed and a quasi-steady model of the forces acting on the ray produced.

\section{From the microscopic to the macroscopic: mathematical analysis and modelling of the trajectories of swimming micro-organisms}

N.A. Hill. Department of Applied Mathematical Studies, The University, Leeds, LS2 9JT

Data on the trajectories of many individual swimming cells, such as Chlamydomonas and $E_{u-}$ glena, have been collected by using a micro-computer based image-processing system. Random walk models have been used to develop techniques for the statistical analysis of the data and to define suitable parameters for the description of the cells' swimming behaviour. These parameters include: (1) a correlation time, which is a measure of how long an individual micro-organism will move in a particular direction before changing its direction of motion; (2) a mean bulk swimming velocity for the whole population of cells; and (3) a bulk diffusivity for the suspension of cells.

These quantities can be used to quantify assays of samples of cells, and they are important in continuum models which describe the macroscopic fluid mechanics of the suspension of cells.

\section{A mathematical model of a rapid starting intermediate Reynolds number undulatory swimmer}

C.E. Jordan. Department of Zoology, NI-15, University of Washington, Seattle, Washington 98195, USA

By combining the full high Reynolds number slender-body theory with a dynamic approximation of the steady-state low Reynolds number resistive theory I model the rapid start of an intermediate Reynolds number undulatory swimmer. The model's predictions of distance travelled with time agree well with observations of rapid starts by the planktonic predator Sagitta elegans (Chaetognatha). With predictions of force generated by the undulating body bounded from above by the maximum isometric stress of invertebrate striated muscle, the model can be used to generate predictions of optimal shape, size and swimming kinematics for a given performance criterion. I have chosen distance travelled over the first $100 \mathrm{~ms}$ of swimming as a biologically reasonable performance criterion. In this case the model predicts a sharp tuning of 
the kinematic parameters, wave speed and wave height to body size in order to maximize performance. In addition, the model predicts increased performance with decreased fin size, due to the increased added mass associated with a larger projected area.

\section{In vivo sarcomere dynamics in the transparent fish Kryptopterus bicirri}

\section{S.M. Kashin, V.R. Edgerton \& R.L. Lieber. Physiological Research Laboratory, Scripps Institution of} Oceanography, University of California at San Diego, La Jolla, California 92093-0204, USA

The diffraction pattern of the laser beam (wavelength $=632 \mathrm{~nm}$ ) passed through the fish body during its swimming was videotaped from the translucent screen. The average sarcomere excursion was $65 \pm 10 \mathrm{~nm}$, representing only 1.8 of a sarcomere length, which is significantly less than was postulated from in vitro measurements. The shortening of sarcomeres occurred 1.4 times faster than their relaxation. The results indicate that the glass catfish myotomal sarcomeres operate with near optimal length (average maximal length $1.88 \pm 0.23$ ) and therefore generate near maximal force.

\section{Functional patterns of swimming bacteria}

\section{J.O. Kessler*, M.A. Hoelzer ${ }^{\dagger}$ T.J. Pedley* \& N.A. Hill*. ${ }^{*}$ Department of Applied Mathematical Studies, The University, Leeds LS2 9JT. 'Department of Anthropology, Columbia University, New York, New York 10027, USA}

The swimming speed and direction of an individual microbe can be affected by various aspects of its immediate environment: chemical concentration, light intensity, shear and gravity. Although there is no direct communication between cells, their concentration can be so great that by their consumption they may collectively modify the properties of every particular individual's neighbourhood. Swimmers may also concentrate themselves; then forces which are insignificant, acting on one cell, can greatly modify the population's dynamics. A mature culture of Bacillus subtilis contains $10^{9}$ cells $\mathrm{ml}^{-1}$, each a swimmer and oxygen consumer, sensitive to the oxygen concentration, and each about $10 \%$ heavier than water. These fundamental elements generate spectacular collective patterns of fluid convection and cell concentration. Beauty and symmetry accompany the inadvertently created patterns which also function to supply oxygen to the entire population.

\section{Comparative locomotor mechanics of the axial skeleton of fishes}

\section{J.H. Long, Jr. Department of Zoology, Duke University, Durham, North Carolina 27706, USA}

Axial skeletons in fishes range in structure from continuous notochordal rods to jointed, mineralized vertebral columns. The goals of this study are (1) to compare the mechanical properties of notochords and vertebral columns and (2) to determine how their locomotor functions differ. I studied the white sturgeon, Acipenser transmontanus, which has a persistent adult notochord, and the blue marlin, Makaira nigricans, which has ossified vertebrae with robust intervertebral connections. I measured the dynamic mechanical properties - angular stiffness, in $\mathrm{Nm} \mathrm{rad}{ }^{-1}$, and damping coefficient, in $\mathrm{kg} \mathrm{m}^{2} \mathrm{~s}^{-1}$ - of these two types of axial skeletons with a bending machine that varies frequency and amplitude. Normalized angular stiffness of the vertebral column of blue marlin is greater than that of the notochord of white sturgeon. The normalized damping coefficient of the vertebral column of blue marlin is higher than that of the notochord of white sturgeon. Thus, the vertebral column offers greater bending resistance and dissipates more energy than the notochord. Greater bending resistance causes (1) increased longitudinal muscle antagonism and (2) increased force transmission in phase with lateral 
displacement. Greater energy dissipation causes (1) decreased elastic recoil and (2) increased force transmission in phase with lateral velocity.

\title{
Are deep-sea 'spiders' six-legged horses?
}

\author{
N.J. Marshall. School of Biological Sciences, Sussex University, Falmer, Brighton, BN1 9QQ \\ C. Diebel \& L.P. Madin. Woods Hole Oceanographic Institution, Woods Hole, \\ Massachusetts 02543, USA
}

Video film (shot from a submersible) and subsequent computer analysis have been used to investigate the swimming movements of four deep-sea munnopsid isopods. These species have not been positively identified, and are referred to as I, II, III and IV. Three modes of swimming have been characterized: 'walking', pedalling and escape.

Munnopsid isopods possess two or three pairs of remarkably long pereopods (nos 2, 3 and 4), and are often observed at depths around $1000 \mathrm{~m}$, hanging vertically with legs spread. As a result they have been nicknamed deep-sea 'spiders'. In species I pereopods 2, 3 and 4 are long and highly setose over their propal segments. With these legs this species 'walks' head-first through the water in a highly unusual manner.

Pereopods 5,6 and 7 are paddle-shaped and possess setae on carpus and propodus. These are used in all species to pedal backwards through the water, although this is rarely seen in species I. The long pereopods of species II, III and IV are not locomotory. Species II often uses only pereopods 5 and 6 to pedal.

In both pedalling and 'walking' the metachronal wave is apparently rostral to caudal and the phase and rhythm remarkable. Instead of using right and left limbs of a segment in synchronous sweeps (e.g. $\left\{\mathrm{R}_{2} \mathrm{~L}_{2}\right\},\left\{\mathrm{R}_{3} \mathrm{~L}_{3}\right\},\left\{\mathrm{R}_{4} \mathrm{~L}_{4}\right\}$, as do many aquatic insects, munnopsid isopods move their pereopods more like a fast walking insect, or an imaginary six-legged horse cantering (e.g. $\left\{R_{2} L_{3}\right\}$, $\left\{R_{3} L_{4}\right\},\left\{R_{4} L_{2}\right\}$ ). Pereopods 5, 6 and 7 (and in I, possibly 2, 3 and 4) are used in synchronous sweeps to elicit the escape response.

\section{Swimming capabilities of Mesozoic marine reptiles: a review}

Judy A. Massare. Department of Geological Sciences, University of Rochester, Rochester, New York 14627, USA

Mesozoic marine reptiles displayed a variety of body shapes and modes of propulsion. Ichthyosaurs, the most highly derived of the marine reptiles, used an axial oscillatory mode. With their deep, streamlined body, narrow caudal peduncle and lunate tail they were probably efficient sustained swimmers. At the other end of the spectrum, marine crocodiles and mosasaurs used an axial subundulatory mode. Their lower hydrodynamic efficiency and elongate shapes must have resulted in relatively slow sustained speeds. They were most likely ambush predators, rather than active pursuit predators like the ichthyosaurs. The crocodiles and mosasaurs had adaptations for rapid acceleration: an elongate, flexible body, a deep caudal peduncle, and long, broad tails.

More problematic are the plesiosaurs, which swam by using two pairs of wing-shaped appendages. Their mode of propulsion combined elements of rowing and flying. The two kinds of plesiosaurs differed in their capabilities. The pliosaurs had more massive limb girdles, more compact bodies and larger, broader limbs than the plesiosauroids. Preliminary calculations suggest that the plesiosaurs were slower sustained swimmers, for a given size, than the ichthyosaurs, but faster than the crocodiles and mosasaurs. 


\section{Escape swimming in the Norway lobster}

P.L. Newland. Department of Zoology, University of Cambridge, Downing Street, Cambridge CB2 3EJ

D.M. Neil. Neurobiology Laboratory, Department of Zoology, Unizersity of Glasgow, Glasgow, G12 8QQ

C.J. Chapman. DAFS Marine Laboratory, PO Box 101, Victoria Road, Torry, Aberdeen, AB9 8DB

We have used a multidisciplinary approach, with methods ranging from neurophysiology to field observations of natural behaviour, to study the rapid backward escape swimming of the Norway lobster, Nephrops norvegicus.

Escape swimming involves a series of rapid power and recovery strokes of the abdomen, termed tail-flips. The initial tail-flip is mediated by giant fibres, and leads to a number of subsequent non-giant flips. The trajectory of the tail-flip depends on the particular giant fibres recruited, and also on the laterality of the stimulus, which influences steering and righting. The duration of swimming appears to be influenced by both neuronal and metabolic factors.

By extending these laboratory studies to field trials, we have confirmed that the swimming performance of Nephrops is similar under natural conditions, and also in interactions between these animals and the trawl nets used to capture them.

\section{The role of fins in the competition between squid and fish}

\section{R.K. O'Dor, J.A. Hoar \& D.M. Webber. Biology Department, Dalhousie University, Halifax, Nova Scotia B3H 4JI, Canada}

The cost of transport for short-finned squid Illex illecebrosus, is five times that of their fish competitors. Loligo pealei, however, with twice the fin area, swims at one-half the cost of Illex at optimum speed. The fin beat frequency of Loligo is higher at low speeds, where they take advantage of energetically favoured undulatory propulsion rather than costly jet propulsion. Since a squid cannot produce fin waves faster than it can move its muscular hydrostat, the fins are most effective at lower speeds. As speed increases, the fin beat frequency decreases and the fins are often rolled up against the mantle, thereby reducing drag and increasing the efficiency of the jet propulsion system. However, the energy costs of carrying an undulatory propulsion system limits the maximum speed and acceleration of the squid. Field studies of the large Azorean Loligo suggest that, in nature, squids behave so as to take advantage of their most economical mode, and allow further economies through 'soaring' in upwelling regions and travelling with the water currents to conserve energy.

\section{Growth and swimming of larval carp}

J.W.M. Osse, J.G.M. van der Boogaart \& A. Terlouw. Department of Experimental Animal Morphology and Cell Biology. Agricultural University, Marijkezeg 40, NL-6709 PG Wageningen, The Netherlands

Webb and Weihs concluded that "as adults and larvae move in the same hydrodynamical regime for important activities, locomotor form and behaviour of all life history stages should be subject to similar physical constraints and form should reflect the effects of analogous selective pressures".

We analysed high speed movies (200 frames s-1) of startle responses of carp larvae of 5.5, 6.1, 10.3 and $20.3 \mathrm{~mm}$ TL to determine the maximum curvature of the body axis. The position of the body axis was reconstructed according to Van Leeuwen et al. The axis was divided into twelve equal segments and angles of curvature between successive segments were measured. At about $30 \%$ body length the $5.5-\mathrm{mm}$ larvae have average maximal angles of curvature exeeding $20^{\circ}$. 
Bigger larvae attain such curvatures only at $50 \%$ body length. Onset and time course of chondrification and ossification of caudal fin ray supporting elements is closely correlated with the change from anguilliform to a more carangiform swimming. The frequency of the tail beats drops from maximal $55 \mathrm{~Hz}$ in $5.5 \mathrm{~mm}$ to $25 \mathrm{~Hz}$ in the $10.3 \mathrm{~mm}$ larvae. The notochord has a thin $(1 \mu \mathrm{m})$ sheath of collagene fibres in the $5.5-\mathrm{mm}$ larvae and some elastine in the horizontal septum. Initial calculations suggest a strain of $2 \%$ in this sheath during swimming.

Although the Reynolds number in the startle responses exceeds values of 200 , the body curvatures in early larvae are reminiscent of resistive swimming which seems to be the regular type of motion in cruising small larvae. No important storage of elastic energy from the deformation of the notochordal sheath seems to occur.

\section{A new thin-walled, pressurized cylinder model for cetaceans}

D. Ann Pabst. Department of Zoology, University of British Columbia, 6270 University Boulevard, Vancouver, British Columbia, V6T 2A9, Canada

Cetaceans can be modelled mechanically as fibre-wound, thin-walled, pressurized cylinders: they are cylindrical in shape, and their axial locomotor muscles are wrapped circumferentially by a collagenous fibre-reinforced membrane, the subdermal connective tissue sheath. The morphology and movements of the cetacean cylinder, though, deviate in measurable ways from that of previous model cylinders. I propose a new functional model, the cetacean as a thin-walled, pressurized cylinder of non-constant, non-circular cross-section that undergoes non-uniform bending along its length. The new model describes some of the forces imposed on such a fibrewound, pressurized cylinder. It also predicts two morphological features of the fibre-reinforced body wall that are required to maintain the cylinder shape during locomotion. As the cylinder tapers, there will be (1) an increase in the angle that fibres describe, relative to the longitudinal axis of the cylinder, and (2) an increase in the number of tensile stays or compressive struts that span the cylinder cross-section. I test the model on bottlenose (Tursiops truncatus) and common (Delphinus delphis) dolphins, using morphological data and mechanical tests of the biaxial tensile behaviour of the subdermal connective tissue sheath. These data support the hypothesis that structural features of the subdermal connective tissue sheath of dolphins are required to maintain their hydrodynamically tuned, external morphology.

\section{Bioconvection}

T.J. Pedley, J.O. Kessler \& N.A. Hill. Department of Applied Mathematical Studies, The University, Leeds, LS2 9JT

Bioconvection, a pattern-forming instability of suspensions of up-swimming micro-organisms that are slightly denser than water, has been recorded since 1848 . The instability mechanism in a shallow suspension, in which the up-swimming causes an increase in cell concentration, and therefore an unstable density stratification, was described in 1961. A uniform suspension of bottom-heavy algal cells has recently been found to be unstable by a different mechanism (gyrotactic instability) which is demonstrated and described.

Theoretical modelling of such suspensions (e.g. of Chlamydomonas nivalis has been put on a rational basis by explicitly accounting for the random distribution of cell orientation by using a Fokker-Planck equation. The dependence of the average cell swimming velocity and the cell diffusivity tensor on the ambient flow field can be computed and compared with observation. Many theoretical problems remain, ranging from the microhydrodynamics of a biflagellate algal cell swimming in a velocity gradient to the nonlinear computations required to explain the form of the observed patterns. 


\section{Neuronal control of swimming in young frog tadpoles}

Alan Roberts, Keith Sillar* \& Kate Boothby. Department of Zoology, University of Bristol, Woodland Road, Bristol, BS8 1 UG * present address: Gatty Marine Laboratory, Department of Biology $\mathcal{E}$ Preclinical Medicine, University of St Andrews, St Andrews, Fife, KY16 8LB

At the time of hatching Xenopus embryos can swim when touched. We have shown that the central nervous system can produce a basic alternating motor drive to the trunk muscles suitable for swimming. Two populations of spinal neurons appear to be responsible. They form a simple reciprocally inhibiting network whose self-sustained rhythmicity depends on rebound from inhibition, mutual re-excitation among excitatory neurons and cellular rhythmicity dependent on the activation of NMDA receptors. The main features of this simple network have been tested by simulation.

Our current work aims to find how this network is turned on, controlled and stopped. Highspeed videos have established that the usual response to touch is to bend away and then swim off. The neural mechanisms were analysed in immobilized tadpoles where neuron activity can be recorded with dye-filled electrodes. The role of spinal interneurons in the initiation of swimming, and in the gating of reflexes during swimming, has been explored. Finally, when tadpoles collide with obstructions they tend to stop swimming. A subclass of head skin mechanoreceptors detect collisions and the pharmacology of such stopping responses implicates a group of brainstem GABAergic reticulospinal neurons in the termination of swimming.

\section{Brainstem control of fin and body movements in swimming dogfish}

B.L. Roberts*, D.H. Paul ${ }^{\dagger}$, R. Williamson ${ }^{\ddagger}$, W. Mos ${ }^{*} \&$ S.J. Timerick ${ }^{\dagger}$. ${ }^{*}$ Department of Experimental Zoology, University of Amsterdam, Kruislaan 320, NL-1098SM Amsterdam, The Netherlands

${ }^{+}$Department of Physiological Sciences, University of Manchester, Manchester, M13 9PT

${ }_{\ddagger}$ Marine Biological Association, The Laboratory, Citadel Hill, Plymouth, PL1 2PB

The basic requirement for swimming "a rhythmical alternation of motor activity that progresses down the body" is met by a program that is generated in the spinal cord but which is then modified by the brain so as to obtain a full repertoire of movement. Cerebral processing implements motor activity via sets of brainstem neurons that connect with the spinal cord. We report here on neuro-anatomical and physiological studies that have shed some light on the operation of these descending systems in relation to swimming in the dogfish Scyliorhinus.

Most of the brainstem neurons that connect with the spinal cord project no further than rostral segments. Descending activity drives myotomal and fin motoneurons both directly, and indirectly via spinal interneurons, and thus (1) evokes fin movements that induce lift, initiate turns and provide postural stability, and (2) modifies and entrains the rhythm of the generating circuits so as to vary tail-beat frequency during cruising locomotion.

\section{Aspects of the design of the fish muscular system}

\section{L.C. Rome. Department of Biology, Leidy Laboratory, University of Pennsylvania, Philadelphia,} Pennsylvania 19104-6018, USA

Fish structure and locomotory behaviour has been viewed principally from a hydrodynamic viewpoint. Additional insight can be gained by assessing these from a muscle viewpoint. In studies of carp and scup, we have elucidated two important constraints. First, fish always use their muscles at near optimal myofilament overlap where maximal force is generated. Second, they use their muscles over a narrow range of $V / V_{\max }$ values $(0 \cdot 17-0 \cdot 36)$ where maximum power and optimal efficiency are achieved $\left(V_{\max }\right.$ is the maximum velocity of shortening and $V$ is the 
shortening velocity during swimming). To enable them to produce all movements, from slow, steady swimming to the escape response, fish have employed a two-gear system: the red muscle (low gear-ratio, low $V_{\max }$ ) and the white muscle (high gear-ratio, high $V_{\max }$ ). The need for fish muscles to work within the constraints noted above largely explains (1) the necessity of the twogear system, (2) the recruitment of red fibres at slow speeds and white fibres at fast ones, (3) the use of burst and coast swimming at very low and at fast speeds, (4) the temperature effects on swimming, and (5) the adaptations in fast swimming species.

\section{Speed changes in the swimming system of the pteropod mollusc, Clione limacina: three gears or two gears with overdrive?}

\section{R.A. Satterlie. Department of Zoology, Arizona State University, Tempe, Arizona 85287-1501, USA}

The pteropod mollusc, Clione limacina, exhibits three forms of swimming: slow, fast and escape. The central pattern generator for slow swimming consists of two groups of antagonistic interneurons that interact through reciprocal inhibitory synapses. The change from slow to fast swimming involves activation of additional interneuron and recruitment of previously inactive motor neurons. Pattern generator reconfiguration allows an increase in wing-beat frequency, while motor neuron recruitment increases the force of wing contractions. This 'change-of-gears' produces a dramatic change in swimming speed.

Escape swimming consists of a ballistic acceleration of swimming followed by a variable period of enhanced fast swimming. A pair of large motor neurons has been identified in each pedal ganglion that (1) innervates the swimming musculature of the ipsilateral wing, (2) produces extremely vigorous contractions of the wing, (3) does not receive any synaptic input from the swim pattern generator, and (4) does not alter activity in any of the identified swim interneurons or motor neurons. Current evidence suggests that these motor neurons may produce initial ballistic response of escape swimming. Since their activity is independent of swim pattern generator activity, it appears as if the escape response does not represent a separate 'gear', but is rather superimposed on fast swimming.

\section{A two-dimensional inviscid model of fish swimming}

W.W. Schultz, Q.-N. Zhou. Department of Mechanical Engineering and Applied Mechanics, College of Engineering, Unizersity of Michigan, Ann Arbor, Michigan 48109-2125, USA

P. W. Webb. Department of Biology, and School of Natural Resources, University of Michigan, Ann Arbor, Michigan 48109-1115, USA

The swimming of neutrally-buoyant fish is simulated by assuming two-dimensional inviscid flow with the wake represented by discrete point vortices. For a fish shape that is a prescribed function of time, we predict the motion of the centre of mass and the orientation angle from conservation of linear and angular momentum. Our fully-computational, two-dimensional model requires no slender-body assumptions nor 'adjustable' parameters.

The computational model compares well to an observed fast-start (acceleration from rest) of a rainbow trout and a rudd turning manoeuvre.

We study effects of body shape and deformation on swimming speed and power for carangiform motions. Steady swimming results show that the predicted maximum speed occurs when the swimming wavelength is equal to the fish length. The velocities are appropriate for observed stride lengths. The simulated swimming power with a simple viscous force model compares favourably to expectations based on measured metabolic rates. Simulations with varying viscous forces suggest that the inviscid wake-induced drag may be as important as, if not more important than, viscous drag. The numerical swimming power shows excellent agreement with trends 
from Lighthill's slender-body theory in the range where the slender-body theory is valid. The effects of the body thickness agrees qualitatively with the conclusions of Newman \& Wu and Newman.

\section{Pleopod movements of a tube-dwelling decapod}

E.J. Stamhuis. Department of Marine Biology, Rijksuniversiteit Groningen, Biologisch Centrum, PO Box 14, NL-9750 Haren AA Gn, The Netherlands

Some marine decapod Crustacea live in a burrow system of tubes in the bottom of the sea. The burrow is periodically flushed by pumping water through the tubes by means of three or four pairs of pleopods to filter the flow for food, or to ventilate the burrow. The pumping of water through narrow tubes can be assumed to be associated with considerable energetic costs. In this paper a study of pleopod pumping movements of the mud shrimp Callianassa subterranea (Montagu, 1808) is presented.

Pleopod movements of animals in perspex artificial burrows or sediment-filled cuvets are described by using high resolution video techniques and computer-aided motion analysis. The forces developed during pumping, the achieved flow speed, the energetic costs and the pumping efficiency are estimated with a simple mathematical model by using quasi-steady-state fluid dynamics for the pleopods and the Hagen-Poisseuille formulas for the flow in the tube. Flow speeds predicted by the model for realistic sets of pleopod parameters are of the same magnitude as measured flow speeds. Mechanical pumping efficiencies calculated from estimated energetic costs are compared to literature values for swimming efficiency in small crustaceans.

\section{Power output of two sizes of Atlantic salmon (Salmo salar L.) at their maximum sustainable swimming speeds}

\section{J.W. Tang \& C.S. Wardle. DAFS Marine Laboratory, PO Box 101, Victoria Road, Torry, Aberdeen, $A B 9$ 8DB}

Maximum sustainable swimming speeds $\left(U_{\mathrm{ms}}\right)$ were determined for two sizes of Atlantic salmon ('large' with BL 0.44-0.45 m, and 'small' with BL $0.15 \mathrm{~m}$ ). For large fish $U_{\mathrm{ms}}=0.91 \mathrm{~m} \mathrm{~s}^{-1}$, for small fish $U_{\mathrm{m} / \mathrm{s}}=0.543 \mathrm{~m} \mathrm{~s}^{-1}$.

Video and cine films of fish swimming close to $U_{\mathrm{ms}}$ were analysed to obtain parameters required for the application of two hydrodynamical models - Lighthill's elongated-body theory and Yates' hydromechanical theory - to determine the mean thrust $(T)$ and power output $(P)$ at these swimming speeds $(U)$. For large fish, Lighthill's model gives $T=0.24 \mathrm{~N}, P=0.21 \mathrm{~W}$; Yates' model gives $T=0.28 \mathrm{~N}, P=0.25 \mathrm{~W}\left(U=0.867 \mathrm{~m} \mathrm{~s}^{-1}\right)$. For small fish, Lighthill's model gives $T=0.0052 \mathrm{~N}$, $P=0.0019 \mathrm{~W}$; Yates' model gives $T=0.0065 \mathrm{~N}, P=0.0024 \mathrm{~W}\left(U=0.37 \mathrm{~m} \mathrm{~s}^{-1}\right)$.

At $U_{\mathrm{ms}}$ it was assumed that all the red muscle was used. Selected fish were frozen and crosssectioned to estimate their red muscle masses. Large fish (740-770 g) had 25-30 g red muscle. Small fish (22-26 g) had 1.1-1.7 g red muscle. A specific power output value of $5-10 \mathrm{~W} \mathrm{~kg}^{-1}$ gave the large fish a capacity of 0.125-0.3 W and the small fish a capacity of 0.007-0.019 W.

Power output values from the different approaches agree well for the large fish $\left(U \sim U_{\mathrm{ms}}\right)$. The small fish were filmed at $U=2 / 3 U_{\mathrm{ms}}$ and this is shown in the differing power figures.

\section{Stone, bone or blubber? Buoyancy control strategies in aquatic tetrapods}

M.A. Taylor. Earth Sciences Section, Leicestershire Museums Service, 96 Nezw Walk, Leicester, LE1 6TD

Control of buoyancy is important to minimize energy wastage and enable slow swimming or hovering. Because of their lungs, tetrapods' buoyancy decreases with depth, but they can inhale 
(at the surface) or exhale to adjust lung volume and depth ('neutral depth') at which they are neutrally buoyant. A theoretical analysis suggests three strategies.

(1) Buoyancy from fat substituted for some lung volume, minimizing total change and rate of change of buoyancy for each depth. It should be best for deep-divers, such as the odontocetes and especially sperm whales (which have very small lungs).

(2) Swallowing stones; rapid attainment and adjustment of neutral depth, useful for shallowwater animals, slow swimmers, and manoeuvring in confined spaces; ignoring accidental ingestion by benthic feeders, stones are found in most underwater 'fliers' (plesiosaurs, penguins, otariids) and crocodilians (ambush predators).

(3) More and denser bone (pachyostosis); less efficient than stone (slows acceleration, metabolically costly). Appropriate for animals grazing on slow or stationary prey at or near the bottom (herbivorous sirenians, walrus Odobenus, and the microphagous reptile Mesosaurus).

\section{Experiments on the neural basis of the rostral-caudal delay during fictive swimming in Xenopus embryos}

Mark J. Tunstall \& Alan Roberts. Department of Zoology, University of Bristol, Woodland Road, Bristol, BS8 1UG

A central feature of anguilliform swimming which is displayed by Xenopus embryos is a rostralcaudal delay in the occurrence of activity in the swimming muscles and, during fictive swimming, in the ventral roots which innervate the swimming muscles. The magnitude of the delay is 3-8 ms $\mathrm{mm}^{-1}$, much smaller than could be accounted for if it depended on axonal conduction velocities and synaptic delays. To try to determine the neural mechanisms involved we have used pharmacological and surgical techniques to look for consequential changes in the magnitude of the rostral-caudal delay. Our experiments suggest that within the central pattern generator there may be a rostral-caudal gradient of both excitation and inhibition. In the latter case we have demonstrated this directly by recording from individual neurons at different spinal levels. A possible implication of this gradient is that rostral neurons may be able to attain their threshold for firing action potentials sooner than more caudal neurons. We consider the ways that rostralcaudal delay could be determined by the nature of this gradient and by the coupling that exists between the component neurons of the spinal pattern generator.

\section{Swimming behaviour of calanoid copepods}

\section{L.A. van Duren. Department of Marine Biology, Rijksuniversiteit Groningen, Biologisch Centrum, PO Box 14, NL-9750 Haren AA Gn, The Netherlands}

A method is presented for the study of swimming behaviour of animals ranging in size from $0 \cdot 1$ to several $\mathrm{mm}$. Through a set of mirrors two orthogonal views of an aquarium are reflected into the lens of a video camera. Parallel infra-red light beams are projected through the aquarium straight into the camera, creating a very sharp image with the animals appearing as black dots against a light background.

Temora longicornis (Müller) is a small North Sea copepod, adults reaching a metasome length of 0.7-1 mm. Over the different stages in their life, from nauplius larvae to adult, they experience a considerable increase in body size and a change in body shape. Escape responses, invoked by administering strong light pulses, are used to measure differences in swimming speeds and patterns. Adults and larger copepodites reached swimming speeds of over $80 \mathrm{~mm} \mathrm{~s}^{-1}(\operatorname{Re}=60)$. The cruising speed of nauplius larvae, however, was a hundred times lower, at Reynolds numbers in the same order of magnitude as measured for swimming ciliates $(R e=0 \cdot 1)$.

Animals swimming at Reynolds numbers between $0 \cdot 1$ and 60 experience an enormous change 
in the influence of viscous to inertial forces on their movements. This causes considerable difficulties in estimating the energetic costs of locomotion.

\section{Fish skin: design for elastic energy storage?}

J.L. van Leeuwen. Neuroregulation Group, Department of Physiology, University of Leiden, Wassenaarseweg 62, PO Box 9604, NL-2300 RC Leiden, The Netherlands

Wainwright et al. have suggested that the helically arranged collagen fibres in the skin of a fish might serve as energy-saving springs in swimming. Alexander calculated that a spring mechanism could work if fibres have angles of pitch below or above the critical angle of zero strain during bending. Alexander's strain calculations (restricted to circular cross-sections) were generalized for arbitrary fish shapes. Fibre orientations down the trunk of the carp (Cyprinus carpio) and the variable fish shape during swimming were used as input parameters. The analysis elucidates important differences in the elastic mechanism along the trunk. During continuous swimming, the fibres act anteriorly as a nonlinear soft spring. Skin stiffness in lateral bending increases towards the caudal peduncle, owing to a decreasing pitch angle of the fibres. Anteriorly, very little strain energy is stored. Posteriorly, the peak strain energy density can be about 50 times higher than anteriorly. Nevertheless, strain energy storage does not seem to be an important energy-saving mechanism in continuous swimming. In intermittent swimming, elastic energy storage is much more important. The increasing stiffness of the skin was also found for the red discus (with a width to height ratio of 0.5 ) and eel. Compared to carp and red discus the eel seems to have a relatively high skin stiffness.

\section{Power output of fish swimming muscle: model and experiment compared}

J.L. van Leeuwen. Neuroregulation Group, Department of Physiology, University of Leiden, Wassenaarseweg 62, PO Box 9604, NL-2300 RC Leiden, The Netherlands

J.D. Altringham. Department of Pure and Applied Biology, The University, Leeds, LS2 9JT

\section{I.A. Johnston. Gatty Marine Laboratory, Department of Biology E Preclinical Medicine, University of St Andrews, St Andrews, Fife, KY16 8LB}

Along the trunk of a continuously swimming fish, muscle fibres are active in different phases during their lengthening-shortening cycle. Therefore, the mean power output changes from positive to negative down the trunk, as modelled for the carp by van Leeuwen et al. An elaborated version of their mechanical muscle fibre model is presented, based on (1) sarcomere ultrastructure, (2) the relation between force and interfilamentary velocity, (3) activation-contraction coupling, and (4) shortening deactivation. The model simulates instantaneous power output for any combination of activation and length change. The accuracy of the predictions was tested against measurements of power output during sinusoidal length changes of isolated fast muscle fibres of the bullrout. The experiments showed a relatively faster deactivation under dynamic circumstances than predicted. A good agreement between simulations and experiments was found, once account was taken of this phenomenon. It was concluded that the model is a valuable tool for predicting the in vivo power output of muscle fibres.

\section{Swimming performance and energetics}

J.J. Videler. Department of Marine Biology, Rijksuniversiteit Groningen, Biologisch Centrum, PO Box 14, NL-9750 Haren AA Gn, The Netherlands

The evolutionary fitness of pelagic fish species largely depends on three aspects of locomotion: speed, endurance and costs of transport. 
Stride length and tail beat frequency are the key parameters determining swimming speed. Stride length has a maximum, species-specific value, reflecting optimum swimming behaviour. The maximum frequency has been predicted from physiological measurements of twitch contraction times and from kinematic data. It is influenced by temperature $\left(Q_{10^{\circ} \mathrm{C}}= \pm 2\right)$ and body length $\left(Q_{10 \mathrm{~cm}}= \pm 0 \cdot 8\right)$. The available maximum values are discussed.

Endurance curves, from the maximum sustained speed up to the maximum burst velocity, have been measured for fish forced to swim in flume tanks, and during spontaneous swimming in pursuit of a moving light pattern in a large circular tank. How good is the match between these data sets?

Costs of transport are calculated from rates of oxygen consumption at different swimming speeds in respirometers. In some cases kinematic data, collected simultaneously, offer the possibility of relating oxygen consumption to stride length and tail beat frequencies.

The aim of this presentation is to examine the available data in search of a consistent picture of the relationships between speed, endurance and costs of transport for as many species as possible.

\section{Design for swimming}

Stephen A. Wainwright. Department of Zoology, Duke University, Durham, North Carolina 27706, USA

In animals that swim by undulating their body and wagging their tail, longitudinal muscle generates the work ( $=$ force $x$ the shortening parallel to the body's long axis) that causes the body to bend. We present the most recent stage in the development of a three-dimensional working model of a king mackerel, Scomberomerus cavalla, as a hypothesis of how the mackerel locomotor system works.

We are concerned with the forceful contraction of muscle and the transmission of this work to the backbone directly and indirectly by pliant collagenous tendon structures. We are also concerned with the forceful swelling of the contractile muscle and the possible function of this work in swimming, perhaps by stiffening the skin, the anterior oblique tendons, and the horizontal and vertical septa, thus making them more efficient elastic energy storers and work transmitters. In the backbone, the bending stiffness of intervertebral joints is the vehicle transmitting the work of the tail wag to the water, and their viscous damping assures the propagation of the body bend to the tail.

A cladogram of animals from prechordate worms through amphioxus, cyclostomes and fishes to the cetaceans is presented, showing information and hypotheses about the step-wise evolution of vertebrate undulatory morphology.

\section{Fish myotomes: their role in supplying force and controlling speed during steady swimming}

\section{C.S. Wardle. DAFS Marine Laboratory, PO Box 101, Victoria Road, Torry, Aberdeen, AB9 8DB}

Recent findings from EMG studies of swimming of mackerel (Scomber scombrus) and saithe (Pollachius virens) compliment findings from kinematic studies. The pulses of force developed by the tail blade are derived from appropriate and precise timing of the activation of particular muscle fibres within each myotome of the lateral muscles. The properties of the muscle and its cross-sectional area varies along the length of the body. At different velocities the pattern of activation ensures there is always one instant of maximum bending moment with a magnitude appropriate to the frequency and with the same myotomes involved in the same phases of the cycle. 
For example, in mackerel (length $0.30 \mathrm{~m}$ ) a maximum frequency of $18 \mathrm{~Hz}$ is maintained by force from the whole cross-sectional area of the white muscle $\left(12 \mathrm{~cm}^{2}\right)$, whereas the maximum sustained frequency of $3.5 \mathrm{~Hz}$ uses only $1 \mathrm{~cm}^{2}$, but the motions are identical.

In contrast the eel, with no tail blade, transfers force to the water as each body section is moved from extreme right to extreme left position and vice versa. Each body section shows a force peak as its lateral velocity reaches a maximum. However the section containing the peak velocity moves continuously from head to tail.

\section{Swimming behaviour and mechanics of a manoeuvrer, the bluegill sunfish (Lepomis macrochirus)}

Paul W. Webb. Department of Biology and School of Natural Resources, University of Michigan, Ann Arbor, Michigan 48109-1115, USA

Bluegill sunfish are temperate representatives of the gibbose-bodied manoeuvring ecomorph. Swimming at sustainable speeds was analysed to (1) test the hypothesis that the high power requirements of flexing self-propulsion result from enhanced frictional drag, and (2) compare swimming behaviour of a manoeuvrer with more commonly studied fusiform species.

Power requirements and power (= drag) coefficients of bluegill were lower than for cruisers such as trout, suggesting that high swimming resistance is not due to enhanced frictional drag. Inertial effects associated with recoil and kinetic energy lost in vorticity are probably more important.

Bluegill used four locomotor patterns at cruising and prolonged speeds: (1) labriform propulsion using the pectoral fins alone; (2) simultaneous labriform and subcarangiform propulsion using the body and caudal fin; (3) subcarangiform propulsion alone; and (4) burst-and-coast swimming. Use of these swimming patterns overlapped. Like other components of the propulsor system, the various swimming patterns differ in power delivery, efficiency, and economy. Adequate power at good efficiency or economy is provided over a wide range of swimming speeds by integrated recruitment of three components of motor systems: (1) muscle; (2) propulsor; and (3) swimming patterns. The greater prevalence of burst-and-coast swimming in bluegill compared to other temperate fishes may relate body volume requirements providing adequate energy for overwintering.

\section{Kinematics and morphology of three locomotor modes in labrid fishes}

M.W. Westneat. Department of Zoology, Duke University, Durham, North Carolina 27706, USA

Fishes of the family Labridae (Perciformes) employ multiple locomotor mechanisms. The goals of this paper are (1) to describe the kinematics and morphology of three locomotor modes that occur in labrids, and (2) to present quantitative models of these locomotor systems that allow prediction of locomotor kinematics. I studied the locomotor mechanisms of lateral undulation, pectoral rowing, and dorsal fin undulation in labrid fishes by using techniques of videography and modelling of locomotor biomechanics. The species Bodianus bilunulatus, Cheilinus unifasciatus, Gomphosus varius and Thallassoma duperrey were studied. Gomphosus and Thallassoma each employ two primary modes of locomotion, whereas Bodianus and Cheilinus use all three modes. Dorsal fin undulations are used for slow cruising, pectoral rowing is used for mid-range speeds, and lateral undulation of the body is used for escape response and capture of elusive prey. The musculo-skeletal systems of the pectoral fin and dorsal fin are modelled by using physical models as well as computer programs based on morphometrics. Morphometric measurements of the pectoral and dorsal fin musculature, skeletal support, and fin rays are the basis for lever 
models of fin ray movements during swimming. By simulating the pattern and extent of muscle contractions, these models accurately predict the observed kinematics of locomotion.

\section{Influence of temperature and ambient oxygen on the swimming energetics of larval cyprinids}

W. Wieser \& R. Kaufmann. Institut für Zoologie, Universität Innsbruck, Technikerstraße 25, A-6020 Innsbruck, Austria

Oxygen uptake $(R(u))$ of the larvae of Danube bleak (Chalcalburnus chalcoides mento) swimming at different speeds $(u)$ is described by the function: $R(u)=\mathrm{R}+\mathrm{a} \mathrm{u}^{\mathrm{b}}\left(R_{\mathrm{s}}=\right.$ standard rate). The increase of oxygen uptake with speed is much steeper at 15 than at $20^{\circ} \mathrm{C}(b=2.03 \pm 0.28$ and $1.67 \pm 0.18$ respectively). As a consequence, swimming performance is diminished, and oxygen debt incurred at the highest speed enhanced, by about 30\% in fish swimming at the lower temperature. Whereas standard and routine respiration rates were well regulated over a $p \mathrm{O}_{2}$-range of from 8.5 to $25.8 \mathrm{kPa}$, the active rates showed a conformer-like pattern, resulting in factorial scopes for activity between two and four. Under hypoxia, the critical swimming speed was lower than under normoxia by about $1.5 \mathrm{l} \mathrm{s}^{-1}$, but the net cost of swimming was also lower by about $30 \%$. On the other hand, hyperoxia neither increased the swimming performance nor did it lead to a further increase of the metabolic cost of swimming. The responses of $C$. chalcoides are discussed in terms of metabolic strategies by which energy-limited animals meet the challenge of environmental deterioration.

\section{The generation of locomotor patterns by the lamprey spinal cord}

Thelma L. Williams. Department of Physiology, St George's Hospital Medical School, University of London, Cranmer Terrace, Tooting, London, SW17 ORE

This talk reviews studies from several laboratories on the mechanisms of locomotor pattern generation in the lamprey. The motoneuronal activity patterns which give rise to locomotion in the intact animal can be produced by the spinal cord in vitro, demonstrating that neither descending commands nor sensory feedback are necessary for stereotypic pattern generation. This pattern-generating ability is distributed along the spinal cord. Mechanoreceptors within the spinal cord mediate sensory feedback to the central pattern generating circuitry. Studies on the in vitro preparation have indicated a role for such feedback in the control of the timing of activation relative to the timing of curvature during swimming in the intact animal. A combined experimental and mathematical study of the lamprey spinal cord viewed as a chain of coupled nonlinear oscillators has indicated some general features of the ascending and descending intersegmental coupling systems.

Computer simulations have provided an initial hypothesis of the circuitry of an uncoupled oscillator, and simulated chains of such oscillators can reproduce some of the major features of the locomotor patterns.

\section{Swimming in bottlenose dolphins (Tursiops truncatus): aerobic and anaerobic limits to performance}

T.M. Williams, W.A. Friedl, M.L. Fong \& J.E. Haun. NOSC Hawaii Laboratory, PO Box 997 Code 511, Kailua, Hawaii 96734, USA

In 1936 Gray introduced a paradox concerning the performance capabilities and physiological limitations of swimming dolphins. The paradox as originally described has been questioned based on theoretical considerations. However, few studies have measured physiological param- 
eters during graded exercise by cetaceans. In view of this, we measured oxygen consumption $\left(\mathrm{VO}_{2}\right)$, respiration and heart rates, and post-exercise blood lactic acid concentration (LA) over a wide range of exercise loads in dolphins. Two Atlantic bottlenose dolphins (mean body mass = $145 \mathrm{~kg}$ ) were trained to push against a load cell at mean force levels of $230 \mathrm{~N}$ to $1800 \mathrm{~N}$. Except for LA, physiological parameters were monitored continuously during exercise bouts maintained for at least five minutes. Results indicated that aerobic metabolism predominated at exercise loads below $340 \mathrm{~N}$ for the female dolphin and $670 \mathrm{~N}$ for the male. Lactic acid concentration remained at baseline levels $\left(22.9 \pm 4.5 \mathrm{mg} \mathrm{dl}^{-1}\right)$ until these 'threshold' loads were exceeded. The animals would not maintain exercise at loads that resulted in $\mathrm{VO}_{2}$ greater than five times resting values or LA 75-102 $\mathrm{mg} \mathrm{dl}^{-1}$ over resting levels. In comparison, dolphins swimming at the routine speeds, 3.8-4.5 $\mathrm{m} \mathrm{s}^{-1}$, demonstrated respiratory rates, heart rates and LA consistent with aerobic performance. Although dolphins are capable of high level, energetically costly performances, these data suggest that routine swimming is relatively inexpensive for these animals.

\section{Some properties of muscle limiting its performance as a power source for swimming \\ R.C. Woledge. Department of Physiology, University College London, Gower Street, London, WC1E 6BT}

During brief periods of activity, the maximum power that can be produced by a muscle is limited by the rate of energy transduction by the actomyosin ATPase system (the crossbridges themselves). This rate is a function of the velocity of shortening of the muscle and shows a clear maximum at about one third of the maximum velocity of shortening. The average power produced during cyclic length changes such as those occurring during swimming, which are approximately sinusoidal, will be less than this optimum value for at least four reasons: (1) the muscle is shortening for only half the time; (2) the velocity during shortening is not always at the optimum value; (3) the muscle cannot be activated instantly at the start of shortening; and (4) the muscle cannot relax instantly at the end of shortening. This last factor is of particular importance in limiting the maximum average power that can be produced. The timing and duration of muscle activation are therefore important in optimizing power output.

If a muscle is to deliver power for more than a small number of cycles, the supply of ATP will become limiting. Under these circumstances, the muscle can deliver more power if it works under conditions (cycle rate and timing of activation) which optimize the efficiency of energy conversion. These conditions are different from those optimizing power output during brief bursts of activity. 


\section{THE THIRD LESLIE COOPER MEMORIAL LECTURE}

\section{Balistiform locomotion}

Professor Sir James Lighthill. Department of Mathematics, University College, London, WC1E 6BT

Balistiform swimming (named after the trigger-fish, Balistes, but occurring rather widely) maintains the fish body in a straight and rigid condition, while achieving propulsive thrust by passing undulatory movements backwards along small but highly flexible dorsal and ventral fins. Yet the observed swimming speed often appears disproportionately large in relation to such small fin movements.

Hydrodynamic analysis suggests two reasons for this: (1) forces generated by small flexible fins attached to a deep rigid body turn out to be very substantially greater than similar fin undulations could possibly produce 'on their own'; and (2) the drag opposing forward motion is very substantially less for a fish body when it moves in a straight and rigid condition than for a body which either $(a)$ is itself engaged in propulsive undulations or $(b)$ responds passively with yawing and side-slipping motions to propulsive motions of the caudal fin.

These considerations taken together could account for a three-fold enhancement of swimming speed. 


\title{
POSTERS
}

\section{Scaling effects on fish muscle fibres performing oscillatory work}

\author{
M.E. Anderson \& I.A. Johnston. Gatty Marine Laboratory, Department of Biology \& Preclinical \\ Medicine, University of St Andrews, St Andrews, Fife, KY16 8LB
}

Fast muscle fibres were isolated from the anterior myotomes of the cod (Gadus morhua L.), and power output determined under conditions simulating steady swimming. Preparations were subject to sinusoidal length changes about in situ resting fibre length. Strain, and the number and timing of stimuli, were adjusted to maximise power output at a range of cycle frequencies. Experiments were conducted at $7^{\circ} \mathrm{C}$ on 46 fish of $10-63 \mathrm{~cm}$ standard length $(S L)$.

The strain required for maximum power output was $\pm 5 \%$ in fish $>20 \mathrm{~cm}$ and $\pm 7-11 \%$ in fish $<20$ $\mathrm{cm}$. For small fish power was at, or near, the optimum over a greater range of cycle frequencies than for large fish. The frequency of oscillations $\left(F_{\text {opt }}\right)$ required for maximum power output decreased with increasing fish length $\left(F_{\text {upt }}=43.65 S L^{-10.51}\right.$, range 5-16 Hz). Peak stress under optimum conditions scaled to $S L^{0.26}$ and $S L^{0,29}$ measured over the 9 th-12th and 2 nd cycles respectively. Power output declined with oscillatory cycle number reaching a steady state by the 10th cycle. Measured over the 2 nd cycle, power output scaled to $B L^{-1.4}$, whereas over the 9 th-12th cycles power output was proportional to $B L^{-0.24}$.

\section{Energetics and power output of isolated fish fast muscle fibres performing oscillatory work}

J.D. Altringham. Department of Pure and Applied Biology, The University, Leeds, LS2 9JT

T.W. Moon. Department of Biology, University of Ottawa, Ottawa, Ontario, K1N 6N5, Canada

I.A. Johnston. Gatty Marine Laboratory, Department of Biology \& Preclinical Medicine, University of St Andrews, St Andrews, Fife, KY16 8LB

Fast myotomal muscle fibres were isolated from the cod (Gadus morhua L.) and the energy cost of contraction measured under conditions simulating swimming. Fibre bundles were subjected to sinusoidal strain changes about in situ length, and stimulated at selected phases in each cycle. Preparations were treated with iodoacetic acid and nitrogen to block ATP synthesis. After an initial decline, force and net work remained steady for up to 64 oscillatory strain cycles. At the end of the last cycle fibres were frozen in isopentane (cooled in liquid nitrogen) and metabolite concentrations determined by HPLC and enzymatic analysis. Concentrations of adenylates did not significantly differ from control values, although a significant increase in IMP concentrations at 64 cycles accounted for the maintenance of relatively high energy charge values. Creatine (C) concentrations increased and creatine phosphate $(\mathrm{CP})$ concentrations decreased, implying a tight coupling of the ATP/ADP reaction to the $\mathrm{CP} / \mathrm{C}$ reaction. Muscle economy (around $12 \mathrm{~mJ}$ net work per $\mu$ mole $C P$ hydrolysed) was independent of the number of work cycles performed, with the exception of 8 cycles $(6.9 \mathrm{~mJ}$ per $\mu$ mole CP). Muscle efficiency values calculated, assuming a Gibb's force free energy change for CP splitting in vivo of $55 \mathrm{~kJ} \mathrm{~mol}^{-1}$, were in the range 12-23\%. 


\title{
Analysis of escape swimming in the mysid Praunus flexuosus by using high-speed video recording
}

\author{
A.D. Ansell. Dunstaffnage Marine Laboratory, PO Box 3, Oban, Argyll, PA34 4AD \\ D.M. Neil. Neurobiology Laboratory, Department of Zoology, University of Glasgow, \\ Glasgow, G12 8QQ
}

In the context of predator-prey relationships, the evolution of fast escape responses has important implications in influencing the effectiveness of predators and the vulnerability, and hence survival, of potential prey. In many marine crustaceans, the escape response comprises a characteristic 'tail-flip' which propels the animal rapidly away from potential predators. Modern high-speed video recording techniques facilitate the analysis of these rapid movements in small crustaceans, and we are using such techniques to resolve details of the escape movements for the mysid Praunus flexuosus (O.F. Muller) and the shrimp Crangon crangon (L.). Results for the former are presented here.

High-speed video recordings of mysids, made in experimental aquaria over a range of controlled temperatures $\left(5-25^{\circ} \mathrm{C}\right)$ have been analyzed by using computer-aided techniques. Key parameters of the escape movements measured include the time course of the escape response and its trajectory, angular velocity and acceleration during flexure and recovery, acceleration and velocity during the backward escape, and the distance travelled. The tail-flip is often preceded by a rotation of the body which serves to direct the trajectory of escape away from the point of stimulus. High-speed video recording has shown the probable involvement of coordinated movements of the antennal scales and uropods in producing this rotation.

\section{Catchability coefficients and fish size: a physiological approach}

Marco L. Bianchini. RAISA/CNR, Via Tiburtina 770, I-00188 Roma, Italy, \& School of Fisheries, University of Washington, Seattle, Washington 98195, USA

In trawls, catchability is generally considered fixed. Conversely, the fact that gear in reality operates differently on different sizes might be understood by intuitive reasoning. Small fish whose maximum speed is low are taken first; the others are herded in front of the net mouth and swim until exhausted. Medium sized fish cannot sustain the speed of the boat for long periods, while larger animals having higher (aerobic) speed and/or higher energy reserves (for anaerobic work) can keep going longer. This study is aimed at demonstrating (analytically and experimentally) that trawl fishing is size-selective, not solely by mechanical causes or avoidance reactions, but mainly because physiological processes of swimming endurance are size-dependent. A new conceptual model taking into account a size-dependent time-lag in the process has been envisaged. While in the classical model, doubling fishing time doubles the catch, it should appear that in reality the doubling of time does more than double the catch. The new mathematical model shows this behaviour, and suggests that the largest size-classes are the ones that substantially contribute to the more-than-proportional-to-time catches. The overall result of the process is similar to fishing the smaller fish longer than the bigger ones.

\section{Swimming of ascidian tadpole larvae}

\section{Q. Bone. Marine Biological Association, The Laboratory, Citadel Hill, Plymouth, PL1 2PB}

The tadpole larvae of the ascidians Ciona and Dendrodoa show essentially the same two movement patterns: symmetrical swimming and asymmetrical tail flicks. Swimming movements at tail-beat frequencies up to $40 \mathrm{~Hz}$ and forward speeds up to $10 \mathrm{~L} \mathrm{~s}^{-1}$ (in the Reynolds number range 5-25) involve large lateral movements of the tail, and large yaw of the trunk. This small-scale 
oscillatory swimming system is very different from that of fish, for the tail movements are produced by muscle cells arranged in three segmented rows along the tail, coupled by gap junctions. Swimming is driven by axons innervating anterior ventral muscle cells. The second type of movement, single or multiple tail flicks, is driven by axons innervating dorsal muscle cells, all of which are innervated along the length of the tail. The middle row of muscle cells are not innervated.

\section{The startle response of the trout: effects of cerebellar lesions}

T.J. Bosch \& B.L. Roberts. Department of Experimental Zoology, University of Amsterdam, Kruislaan 320, NL-1098SM Amsterdam, The Netherlands

The effect of partial cerebellectomy on the startle response (SR) of the trout, Oncorhynchus mykiss, was investigated with high-speed film, video analysis and EMG-recordings from white muscle. Surgery was performed under MS-222 anaesthesia; 150 SRs from 20 fish were analysed.

A typical biphasic response is seen in normal, lesioned and sham fish. The first phase, the Cbend ( $20-100 \mathrm{~ms}$ ), is highly stereotyped for the first $15-20 \mathrm{~ms}$. The tail-flip is more variable and lasts about $70 \mathrm{~ms}$. The trajectory is more variable in lesioned fish. The transition between the two phases is immediate, but in lesioned fish sometimes 20-60 ms delayed. The EMG-recordings show a large spike at the onset of phase 1, followed by a variable number of smaller spikes, and subsequently a smaller amplitude activity contralaterally (phase 2). The interval between activities on each side is $10.3 \pm 3.0 \mathrm{~ms}$ in normal and $21.6 \pm 12.3 \mathrm{~ms}$ in lesioned fish.

It thus seems that the cerebellum has no role in the first rapid phase of the response, but does coordinate the onset and execution of the tail-flip.

\section{Repetitive stimulation of the squid giant axon exerts graded control over mantle tension}

Euan R. Brown* \& Quentin Bone. Marine Biological Association, The Laboratory, Citadel Hill, Plymouth, PL1 2PB. *also Physiology Group. Biomedical Sciences Division, King's College, London, WC2R 2LS

The giant axons of the squid were thought to control the escape response by delivering a single action potential to the mantle muscle. Here the effects of repetitive stimulation of the giant axon on mantle tension are reported.

Lively squid (Alloteuthis subulata) were decapitated and the most medial third order giant axons were selectively stimulated electrically at a range of frequencies. Surface action potentials and mantle muscle tension were recorded (mantles were bathed in oxygenated Hepes buffered artificial sea-water) and it was found that significant increases in twitch tension could be generated in the mantle by repetitive stimulation $(3-7$ action potentials at $30-70 \mathrm{~Hz}$ ). These results accord well with volleys of action potentials recorded from the giant fibre system during escape jetting and suggest that squid can vary the magnitude of the mantle twitch by varying the number and frequency of action potentials delivered to the mantle via the giant fibres.

\section{Physiological properties of squid mantle muscle bundles}

Euan R. Brown ${ }^{* \ddagger}$, Malcolm L. Usher ${ }^{\dagger} \&$ Quentin Bone*. ${ }^{*}$ Marine Biological Association, The Laboratory, Citadel Hill, Plymouth, PL1 2PB. ${ }^{+}$Department of Physiology, University College, London, WC1E 6BT. ‘also Physiology Group, Biomedical Sciences Division, King's College, London, WC2R 2LS

In the squid mantle there are several distinct muscle types which operate to produce jetpropelled swimming. Here we report some properties of isolated mantle muscle bundles. 
Squid (Loligo forbesi) were decapitated and muscle bundles from the central region of circular mantle muscle dissected out in chilled artificial sea-water (ASW) and stimulated electrically while tension was recorded via a transducer. Twitch-like responses from strips were reversibly abolished by incubation in nominally $\mathrm{Ca}^{2+}$ free ASW. Iontophoretic filling of individual muscle fibres with Lucifer yellow dye (5\%, MW 521.6) revealed that muscle fibres were strongly dyecoupled and could be uncoupled by incubation in $10 \mathrm{mM}$ octan-1-ol ASW (lower concentrations failed to uncouple the cells).

The results indicate that these muscle bundles require extracellular $\mathrm{Ca}^{2+}$ to contract and that as the muscle fibres are coupled, only a limited number would need to be innervated.

\section{Swimming speeds of large pelagic fish}

Francis G. Carey. Woods Hole Oceanographic Institution, Woods Hole, Massachusetts 02543, USA Barbara A. Block. Department of Anatomy, University of Chicago, 102557 th Street, Chicago, Illinois 80637, USA

Jill C. Scharold. State University of California, Fullerton, California 92634, USA

Swimming speeds of sharks, billfish and tunas have been measured both as translation in position over periods of many hours and by direct telemetry of swimming speed. Both methods agree in showing that sharks, tunas and marlins swim in a slow and steady fashion. There are occasional 10-30 s bursts of speeds up to $200 \mathrm{~cm} \mathrm{~s}^{-1}$ or so, but the range is usually $40-100 \mathrm{~cm} \mathrm{~s}^{-1}$. Tail beat measured directly from a sensor on the caudal peduncle, and indirectly from regular periodic oscillations in the speed sensor, show that the fish have a slow, steady, very regular tailbeat: it is not stroke-stroke-glide. The large fish, $>2 \mathrm{~m}$ body length, often move at the same speeds as cod. Over weeks of telemetry records, we have seen no evidence for the very high speeds commonly mentioned in the literature and believe that such high-speed bursts are quite rare events. The swimming speeds from telemetry experiments are similar to those estimated from tag returns for ocean-crossing fish.

\section{Do whales extract energy from ocean waves?}

\section{K.C. Curren, N. Bose \& J. Lien. Whale Research Group, 230 Mount Scio Road, Memorial University of Newfoundland, St John's, Newfoundland A1B 3X5, Canada}

It has been suggested that the flukes of cetaceans are capable of absorbing energy from ocean waves while swimming. Depending on the length of the whale and wave conditions, propulsive power savings may be great enough to be significant in the energy budget of whales.

Examination of the movements of satellite-tagged whales when the wave conditions are known, are a means of testing whether whales extract energy from waves. Complete morphometrics, a 90-day satellite track and corresponding wave conditions were available for a long-finned pilot whale (Globicephala melaena) swimming off the coast of the eastern United States. Statistical analysis was performed to determine if a correlation existed between periods when large distances were covered and optimal wave conditions were prevalent. 


\title{
Power and efficiency of isolated muscle fibres moved and stimulated to mimic locomotion
}

\author{
N.A. Curtin. Department of Physiology, Charing Cross \& Westminster Medical School, \\ Fulham Palace Road, London, W6 8RF \\ R.C. Woledge. Department of Physiology, University College London, Gower Street, \\ London, WC1E 6BT
}

Bundles of white myotomal muscle fibres from the dogfish, Scyliorhinus canicula, were stimulated during sinusoidal length changes chosen to mimic what occurs during swimming. Force and heat production were measured. The amplitude of movement was about $10 \%$ of the fibres' rest length, and a burst of three stimuli was given in each mechanical cycle at $12^{\circ} \mathrm{C}$. The frequency of the mechanical cycle and the timing of the period of stimulation were varied systematically to find conditions that gave the maximum mechanical power output (calculated as the net work in a complete mechanical cycle divided by the cycle time), and the maximum efficiency (the ratio of net work to total energy output). The cycle frequency giving maximum power was about twice that giving maximum efficiency. These results show that when muscle is operating to give the fastest swimming, its efficiency of energy conversion is compromised.

\section{Kinematic analysis of amphibian tadpole swimming}

N.C. Dale \& S.R. Soffe. Department of Zoology, University of Bristol, Woodland Road, Bristol, BS8 1UG

We describe a method for kinematic analysis of anguilliform movement in amphibian tadpoles. Spatial coordinates are ascribed to a series of 11 equidistant points along the whole body midline, by using selected frames from the sequence to be analysed. We can then compute a variety of measures for each point including: (1) angles of bending; (2) lateral displacement; and (3) forward displacement. From any of these we can compute cycle period, longitudinal phase delay and a measure of symmetry in the movement by fitting sinusoidal functions to the data. We have applied this analysis to high-speed video recordings of swimming in Rana embryos. The nature of the travelling wave that underlies swimming appears different according to which approach is used. From (1), the phase delay appears larger along the middle of the animal and smaller towards each end with a total phase delay of approximately 0.7 . From (2), the total phase delay is approximately 1.0 with the largest phase delay at the level of the rostral trunk. Compared to their lateral displacements, bending around each point may depend more upon local forces and less upon movement of distant body parts. Thus bending angles may relate better to underlying neuromuscular activity. In support of this, total phase delay in motor discharge recorded electrophysiologically is approximately $0 \cdot 6$.

\section{Forces generated during locomotion can influence intracranial pressure in bluefish (Pomatomus saltatrix)}

\author{
A.B. DuBois*, S.H. Fox ${ }^{\dagger} \&$ C.S. Ogilvy*. *John B. Pierce Laboratory, 290 Congress Avenue, \\ New Haven, Connecticut 06519, USA \\ ${ }^{\dagger}$ Neurosurgical Service, Massachusetts General Hospital, Boston, Massachusetts, USA
}

Bluefish weighing $2 \mathrm{~kg}$ can accelerate at a rate $3 \mathrm{~g}$, reaching a speed of $5.5 \mathrm{~m} \mathrm{~s}^{-1}$. The thrust of 6 $\mathrm{N}$ is then opposed by equal drag force. This report concerns the way that forces of locomotion affect the vertebral column, spinal canal, intracranial fluid pressure, and possibly cerebral blood flow of bluefish. Isolated vertebral bodies were shortened by $8 \%$ of initial length when compressed by $50 \mathrm{~kg}(490 \mathrm{~N})$. Nine vertebrae attached to each other were shortened by $1 \%$ by a mass of $6 \mathrm{~kg}$ 
(59 N). A spinal canal $45 \mathrm{~cm}$ long would have a cross-sectional area of $0.1 \mathrm{~cm}^{2}$. If it were compressed by $59 \mathrm{~N}, 0.045 \mathrm{ml}$ of spinal fluid would be displaced into the cranial vault. From pressure-volume curves of the skull we estimate that this would increase intracranial pressure almost 60 torr. Indeed, longitudinal compression of the body by using a $2.7 \mathrm{~kg}$ mass on a pulley increased the intracranial pressure by 20 torr. Bending the spine $45^{\circ}$ to the side increased intracranial pressure by 16 torr. Flexing the peduncle from side to side during longitudinal compression with a mass of $2.7 \mathrm{~kg}$ raised the intracranial pressure to 48 torr. Finally, intracranial pressure fluctuations of \pm 20 torr were measured in the skull of a bluefish swimming at $1.1 \mathrm{~m} \mathrm{~s}^{-1}$. We conclude that thrust and drag coupled with flexing of the spine during locomotion can influence intracranial pressure, and indirectly oppose the increase of cerebral blood pressure that must occur during swimming.

\section{The role of thin filaments in morphogenetic change prior to litholophus swimming}

J. Febvre \& Colette Febvre-Chevalier. Université de Nice, F-06034 Nice, France \& Laboratoire de

Biologie Cellulaire Marine, URA 671 CNRS, Observatoire Océanologique de Villefranche-sur-Mer, F-06230 Villefranche-sur-Mer, France

The acantharians are pelagic protozoans. During their vegetative life the cell body is approximately spherical. It is centred around a mineral skeleton made up of 20 radial needle-shaped spicula of strontium sulphate. An outer pellicle, called the periplasmic cortex, is anchored to the tip of the spicula via myonemes. The myonemes consist of contractile bundles of nonactin filaments involved in buoyancy regulation. They show an alternation of thin dark bands and long clear zones. The periplasmic cortex is made up of a patterned network of thin filaments and looks like a patchwork assembled by elastic junctions.

In the order Chaunacanthida, gametogenesis occurs within a cyst after modification of the motile behaviour and important morphogenetic change. Within a few minutes the spicula turn around the cell centre, resembling a closed umbrella. This new form, designated the 'litholophus', propagates forwards slowly. During this movement the outer structures (cortex and myonemes) and the spicula are shed backwards. The ultimate result of this transformation is an oval cyst within which rapid nuclear fission and meiosis occur. The cyst is covered with polygonal scales and bears small pores and large pits from which gametes spread out later.

Thin sections through the cell centre of the vegetative stage and litholophus form show that the bases of the spicula are embedded in a dense fibrillar matrix. This substance consists of thin filaments (2-4 $\mathrm{nm}$ in diameter) that are lying between the spicula and are anchored to their surface. Such a litholophus transformation may involve this nonactin filament system. In addition to the filament-based matrix, a reticulate substance of unknown composition and large lipid droplets are generally found in the cell centre. Litholophus transformation can be reproduced artificially with $\mathrm{Ca}^{2+}$ blockers and poisons of ATPases.

\section{Factors affecting swimming ability and its recovery in the Norway lobster (Nephrops norvegicus)}

R.H. Field, A.C. Taylor, D.M. Neil. Neurobiology Laboratory, Department of Zoology, University of Glasgow, Glasgow, G12 8QQ

\section{C.J. Chapman. DAFS Marine Laboratory, PO Box 101, Victoria Road, Torry, Aberdeen, AB9 8DB}

Nephrops norvegicus, like other lobsters and crayfish, react to threatening stimuli by production of the tail-flip escape response. The rapid tail flexions involved are produced by the deep abdominal musculature. Enzyme histochemical techniques have been used to show this musculature to be fast phasic in type, with high ATP-ase activity and little capacity for oxidative 
phosphorylation. Studies of the anaerobic metabolism and recovery potential of these muscles have been undertaken to determine whether the cessation of swimming after repeated stimulation (so-called 'functional exhaustion') is due to metabolic exhaustion of inhibition, or to habituation to stimuli eliciting the response. Animals were tested for their response to repeated, standard, localized stimuli, eliciting lateral giant-fibre mediated swimming bouts. Results show that recovery of full swimming ability is linked to the recovery of near normal muscle and haemolymph concentrations of L-lactate. Concentrations of the immediate and secondary energy supplying compounds ATP and D-glucose in tail muscle appear to be non-limiting to escape swimming. Recovery potential is also prejudiced by the degree of localization of stimulation, higher localization leading to reduced recovery of swimming ability. These results indicate that cessation of, and recovery from, escape swimming in $N$. norvegicus is influenced by both metabolic and neuronal factors.

\title{
Septal structure and function in fish muscle
}

A.R. Gardner-Medwin. Department of Physiology, University College London, Gower Street, London, WC1E 6BT

\section{N.A. Curtin. Department of Physiology, Charing Cross \& Westminster Medical School,} Fulham Palace Road, London, W6 8RF

The septal tendon sheets separating myomeres in fish muscle usually lie at an angle to the fish axis, often forming nearly circularly symmetrical cones. The muscle fibres sometimes, but not always, run at an angle to the fish axis. Analysis of the stresses to be expected in a bending fish suggests that there are two independent problems that the arrangement of fibres and tendons may help to overcome. First, where the muscle fibres lie at an angle, the constraints on distortions of the tissue imposed by the conical tendon sheets can provide a mechanical advantage, whereby the overall muscle block shortens by a smaller percentage of its length than the individual muscle fibres. This increases the longitudinal force per cross-sectional area. Second, tendons between the skin and the backbone may serve to keep the fish thin when it bends. These must run at less than $45^{\circ}$ to the axis to avoid restricting the volume under the skin on the inner side of a bend and preventing the muscle blocks from shortening and doing work.

\section{Can swimming performance be predicted from statocyst morphology in cephalopods?}

\author{
W. Glanfield. University College of North Wales, Bangor, Gwynedd
}

A. Witkam. Agricultural University, Marijkeweg 40, NL-6709 PG Wageningen, The Netherlands

R. Williamson. Marine Biological Association, The Laboratory, Citadel Hill, Plymouth, PL1 2PB

It has been argued that the locomotory performances of both vertebrates and cephalopods can be correlated with the sensory response characteristics of their relative vestibular systems. Thus, it may be possible to predict the swimming performance of an animal from a morphological examination of its vestibular system. As an initial test of this hypothesis for cephalopods, we have determined the morphometric changes in cuttlefish statocysts with increasing animal size.

At hatching the statocysts of Sepia officinalis are relatively large, but they do not increase proportionately with mantle length. With increasing animal size there is a correlated increase in both the length of a statocyst crista segment as well as the number of hair cells in a segment. There is also an initial increase in the number of rows of hair cells in a crista segment, but no apparent increase in hair cell size. 
From these data we can infer that afferent sensitivity of the statocyst, particularly at lower frequencies, increases with animal size, presumably reflecting a better control of manoeuvring.

\section{Convergent flow on the caudal fin during undulatory locomotion}

J. Glasheen. Department of Organismal and Evolutionary Biology, Harvard University, Cambridge, Massachusetts 02138, USA

R. Blickhan \& W. Nachtigall. Fachrichtung Zoologie, Universität des Saarlandes, D-6600 Saarbrücken, Germany

Current hydrodynamic theories assume that the caudal fin of a fish swims in undisturbed fluid. Our movements reveal that undulatory movement forms a convergent flow. At mid-stroke the oncoming flow strikes the caudal fin at an angle of about $16^{\circ}$, while the flow on the other side is approximately parallel and slower. Assuming circulation theory for lift production, the indicated flow seems to be disadvantageous.

We investigated this question by stationary simulation of the instantaneous pattern of the nonstationary flow in a wind tunnel. A flat plate served as a model for the fin, flow deflectors and speed modulators formed the desired flow pattern.

Lift $(L)$ and drag $(D)$, as measured during convergent flow, were almost halved as compared to the free-flow condition; the lift to drag ratio, however, was only slightly reduced for equivalent flow speeds (convergent $L / D=3 \cdot 73$, non-convergent $L / D$ ratio $=4 \cdot 01$ ). Moreover, the convergent flow set-up resulted in a much higher angle of separation $\left(40^{\circ}\right)$ than is found without convergent flow $\left(20^{\circ}\right)$. However, this advantage does not seem to be exploited by the fish, whose angles of attack rarely exceed $18^{\circ}$. The results suggest that the resulting flow directions may partially compensate for an otherwise adverse speed distribution.

\section{Swimming speed and muscle activation in rainbow trout}

A.B. Kesel, R. Blickhan \& W. Nachtigall. Fachrichtung Zoologie, Universität des Saarlandes, D-6600 Saarbrücken, Germany

The kinematics of fast locomotion in fish are characterized by an undulatory wave travelling caudally at a speed $(V)$ which is slightly higher than the speed of locomotion $(U)$. This wave is produced by a corresponding wave of muscle activation whose speed $\left(V_{E M C}\right)$ is considerably higher than $V$. No information is available on how the relationship between kinematics and muscle activation is affected by swimming speed.

Trout swimming freely in a water tunnel were used to investigate muscle activity at various positions along the body $(0.4 \mathrm{~L}$ to $0.7 \mathrm{~L})$ and various depths $(5 \mathrm{~mm}$ to $10 \mathrm{~mm}$ ) relative to swimming speed $\left(1.5 \mathrm{~L} \mathrm{~s}^{-1}\right.$ to $\left.2.5 \mathrm{~L} \mathrm{~s}^{-1}\right)$.

Whereas $V$ remains constant along the animal's body $V_{\mathrm{EMC}}$ decreases by a factor of four or five as the activation wave travels caudally. It is remarkable that $V_{\mathrm{EMG}}$ is independent of swimming speed whereas the speed of the undulatory wave changes proportionally to the swimming speed. An increase in speed is achieved by increasing frequency of muscle activation, reducing duration of activity and increasing the depth of muscle recruitment $\left(5 \mathrm{~mm}\right.$ at $2 \mathrm{~L} \mathrm{~s}^{-1} ; 10 \mathrm{~mm}$ at $U>$ $\left.3.5 \mathrm{~L} \mathrm{~s}^{-1}\right)$. The absolute delays of muscle activation between various positions are independent of swimming speed. 


\title{
Flow analysis supported by image processing
}

\author{
C. Krick, R. Blickhan \& W. Nachtigall. Fachrichtung Zoologie, Universität des Saarlandes, \\ D-6600 Saarbrücken, Germany
}

Only a small amount of data is available on the flow pattern in the vicinity of freely swimming fish, and none of the methods applied so far have allowed the quantification of flow velocities. By filming the path of small, suspended particles local flow velocities can be determined. We developed an interactive image-processing technique which allows for a fast and critical data screening and evaluation.

The pattern of the flow within a plane selected by illumination with a light knife, is recorded on video tape. An image sequence of interest is then stored in a frame buffer of an image-processing board. The data can be reduced by high pass and grey scale filtration. A programmed cine-loop and a long time-exposure generated by overlays facilitates screening of the data. Distances, speed, and angles can be measured directly on the screen with mouse assistance. Particles are traced automatically in a sequence of pictures to determine the local velocity. From the line integral along a specified circle around a vortex core its circulation can be calculated.

\section{New functional aspects of fish skin during locomotion}

U.K. Müller, R. Blickhan. Fachrichtung Zoologie, Universität des Saarlandes, D-6600 Saarbrücken, Germany

R. McN. Alexander. Department of Pure and Applied Biology, The University, Leeds, LS2 9JT

Fishes have crossed helical fibres in their skin. This design feature cannot be explained in terms of protection or adjustment to the environment but probably serves to transmit muscle forces during locomotion. This demands a reasonably high stiffness of the skin.

To measure the strain along the fibres as the fish swims, strain transducers were fixed on the skin. The experiments reveal that the strains oscillate according to the undulation of the body and increase from 0.01 to 0.03 for swimming speeds from 0 to $5 \mathrm{~L} \mathrm{~s}^{-1}$. This resembles Alexander's predictions for bending a fibre-wound cylindrical body. The corresponding stresses calculated for a Young's Modulus of $40 \mathrm{MPa}$ range from 0.4 to 1.12 MPa.

In contrast to the stress, the curvature of the skin along the body axis decreases with increasing swimming speed. It is reasonable to interpret the stresses at low speeds as induced by the body curvature. According to this assumption at least $80 \%$ of the stress at $5 \mathrm{~L} \mathrm{~s}^{-1}$ must be due to an additional force other than curvature. This corresponds with Wainwright's idea of the fish skin as an exotendon.

\section{Muscle function in the fast swimming scup}

\section{L.C. Rome, I. Choi, A. Sosnicki \& G. Lutz. Department of Biology, Leidy Laboratory, University of} Pennsylvania, Philadelphia, Pennsylvania 19104-6018, USA

Electromyography shows that scup can swim to a maximum speed of $80 \mathrm{~cm} \mathrm{~s}^{-1}$ with their red muscle whereas previous results showed carp can swim only to $45 \mathrm{~cm} \mathrm{~s}^{-1}$. Our aim was to evaluate the adaptations that enable scup to swim nearly twice as fast as carp. Although we anticipated that at their respective maximum speeds the muscle of scup would be shortening at twice the velocity $V$ of the carp, we found $V$ to be equal ( 2.04 muscle lengths $\left.s^{-1}\right)$. At their respective maximum speeds, the $26 \%$ higher tail-beat frequency in scup is balanced by a $28 \%$ lower sarcomere excursion, giving $V$ equal to that of the carp.

The smaller sarcomere excursion in scup is primarily associated with a less undulatory style of swimming, with smaller backbone curvature. This may be an important adaptation which not 
only reduces $V$, but may also reduce drag.

We also found that the maximum velocity of shortening $V_{\max }\left(5.63\right.$ muscle lengths $\left.\mathrm{s}^{-1}\right)$ was nearly the same as that of carp. Thus the red muscle of scup shorten at the same velocity $V$ and over the same narrow range of $V / V_{\max }(0.017-0.36$, i.e. where maximum force is generated) as carp. Therefore $V / V_{\max }$ appears to be an important design constraint.

\section{Endurance and oxygen consumption during intermittent locomotion of fish}

\section{E. Schlessmann, R. Blickhan \& W. Nachtigall. Fachrichtung Zoologie, Universität des Saarlandes, D-6600 Saarbrücken, Germany}

Burst and glide swimming can reduce the cost of locomotion by about $30 \%$ depending on drag and the efficiency of sustained, i.e. aerobic, locomotion. During intermittent terrestrial locomotion of humans and crabs the observed increase in endurance can be attributed to partial recovery in the pauses from anaerobic sprints.

To investigate whether similar results could be successful in fish locomotion, we compared endurance and oxygen consumption during intermittent exercise $\left(U=2.84 \mathrm{~L} \mathrm{~s}{ }^{-1}, 1-3 \mathrm{~min} ; U=2.28 \mathrm{~L}\right.$ $\mathrm{s}^{-1}, 1-3 \mathrm{~min}$ ) and during steady-state locomotion in rainbow trout swimming in a water tunnel.

No difference could be found, partially due to the large intra-individual variability of endurance and oxygen consumption. Based on the considerable differences in the time constants of the recovery process (ATP, PCr, lactate, glycogen) between fish on the one hand and humans and crabs on the other, as well as on the different speed relationship of metabolic cost, the selected intervals should not allow for sufficient recovery. Nevertheless, our results show that stamina does not suffer during intermittent locomotion.

\section{Mechanics of escape jetting in the large squid Loligo forbesi}

\section{R.E. Shadwick. Physiological Research Laboratory, Scripps Institution of Oceanography, University of California at San Diego, La Jolla, California 92093, USA}

Simultaneous measurements of jet pressure and external diameter were made on large specimens of $L$. forbesi (1.2-2.7 kg) while jetting unrestrained in an aquarium. Pressures were measured by a strain gauge transducer linked to a mantle cavity cannula and recorded on a strip chart. Mantle diameter was measured from video images recorded through the bottom of the aquarium. Pressure and diameter waveforms were digitized and analysed according to Gosline \& Shadwick.

Escape jet pressures ranged from 30 to $45 \mathrm{kPa}$, nearly double those recorded previously in smaller squid. The jet contraction phase lasted about $400 \mathrm{~ms}$, with peak pressure occurring in the first 100-120 ms. Hyperinflation of the mantle prior to escape jetting was often observed. Sequential jets occurred as rapidly as $1 \mathrm{~Hz}$ while resting respiration rates were only $0 \cdot 5-0 \cdot 6 \mathrm{~Hz}$. Comparison of the hydrodynamic work done by the circular muscles to the maximum energy available from the muscular contraction gives a term describing the 'efficiency' of muscle utilization in producing the jet thrust. In the latter half of the jet cycle the hydrodynamic work drops rapidly and a substantial portion of muscular output appears to be available to deform the mantle collagen framework, a structure previously shown to function in elastic energy storage. Based on this assumption, the compressive stress-strain curve for the mantle was calculated and shown to agree with previous measurements in much smaller squid. 


\section{Development of swimming in frog tadpoles}

\section{K.T. Sillar, J. Wedderburn \& J. Simmers. Gatty Marine Laboratory, Department of Biology \&} Preclinical Medicine, University of St Andrews, St Andrews, Fife, KY16 8LB

The hatchling Xenopus embryo swims by lateral undulations of the trunk and tail at $10-20 \mathrm{~Hz}$. Swimming results from the activity of a neuronal network in the spinal cord and brainstem which can operate in the absence of sensory feedback. The swimming rhythm consists of a single impulse in motoneurons on each cycle of activity. Activity alternates on opposite sides and progresses rostrocaudally down the body.

We have examined the subsequent development of the swimming rhythm in immobilized Xenopus tadpoles during the first day of larval life. Within this period motor activity recorded in ventral roots changes from a brief, 5-6 ms impulse on each cycle, to bursts of discharge lasting around $20 \mathrm{~ms}$. The motor rhythm becomes more variable so that burst durations vary on a cycle by cycle basis and the frequency range over which swimming can occur extends. Intracellular recordings from spinal neurons during swimming show that they acquire an ability to fire multiple impulses shortly after hatching and that the synaptic input they receive during swimming increases in complexity. Our current work investigates which changes in the nervous system are responsible for the development of a simple embryonic rhythm into a more robust and flexible larval form.

\section{Acceleration during S-starts in a water tunnel}

\section{A. Simons, R. Blickhan \& W. Nachtigall. Fachrichtung Zoologie, Universität des Saarlandes,} D-6600 Saarbrücken, Germany

To overcome body drag and inertia, the undulatory wave is used in both steady-state locomotion and in S-starts. While swimming in a flume, acceleration towards a prey must be achieved in addition to the work necessary for sustained swimming. We investigated whether this could result in a limitation of the ability to accelerate from various speeds.

While swimming in a water tunnel, rainbow trout were trained to accelerate towards a needle dipped into the water at a distance of $0.5 \mathrm{~m}$. The behaviour was recorded at 200 frames $\mathrm{s}^{-1}$ with a $16 \mathrm{~mm}$ high-speed camera.

All starts showed a stereotype pattern: after a phase of acceleration achieved by increasing tailbeat-amplitude $(0.16 \mathrm{~L})$ and frequency $(30 \mathrm{~Hz})$, the animals glided towards the prey. The maximum speeds achieved ranged from 5.5-11 $\mathrm{L} \mathrm{s}^{-1}$.

The observed lack of dependency on speed can be explained by the dominance of inertial forces. We do not know whether the animals accelerated at maximum effort. Nevertheless, the achieved accelerations (up to $39 \mathrm{~m} \mathrm{~s}^{-2}$ ) are similar in magnitude to the values reported for C-starts in escape situations.

\section{Forces in tethered trout}

\section{F. Truxa, R. Blickhan \& W. Nachtigall. Fachrichtung Zoologie, Universität des Saarlandes, D-6600 Saarbrücken, Germany}

The basic hydrodynamic mechanisms of propulsion in fish are well understood. In contrast, it is still unknown how forces are transmitted from muscle fibres to the environment. We approached this question by measuring the lateral forces along the animal's body under isometric conditions.

The anaesthetized rainbow trout $(L=23 \mathrm{~cm})$ was suspended within a series of five specially designed force transducers. The signals were sampled online at a rate of 200 samples $\mathrm{s}^{-1}$ for each channel. 
As the animal tried to escape after recovering from anaesthesia, especially high forces were observed. The measurements revealed a force maximum ( $c a 9.5 \mathrm{~N}$; tail-beat frequency $9 \mathrm{~Hz})$ in the peduncular region $(c a 0 \cdot 7-0.8 \mathrm{~L})$. The forces are about twice the values published for a onesided myotomal preparation where the musculature of the anaesthetized fish was stimulated electrically. The force maximum is behind the site of maximum muscle cross-sectional area indicating force transmission via connective tissue or skin.

\section{The effect of oscillating bodies on the flow}

Bodo E. Ungerechts. Olympiastützpunkt Hannover/Wolfsburg, Ferdinand-Wilhelm-Fricke-Weg 10 , D-3000 Hannover 1, Germany

Discussions of the propulsive forces produced during swimming uncover our lack of information concerning the flow effects along bodies which are not rigid but which change their form periodically. In most cases the flow is assumed to be stationary, and this leads to a large discrepancy between the calculated external forces and the muscular forces. In response some hypotheses have been advanced about the reduction of flow resistance, but these do not solve the paradox. The purpose of this paper is to investigate the effect of oscillating bodies on the flow.

The reaction of the flow to acceleration by the oscillating body is determined by measuring the hydrodynamic pressure acting normal to the surface of models of fast-swimming vertebrates. To this end the flexible models were put in a water tunnel and the swimming movements were generated by a specially made apparatus.

The findings demonstrate a pressure distribution along a rigid body in agreement with Bernoulli's Theorem. When set in motion at different frequencies and different flow speeds the pressure distribution also starts oscillating. In stationary flow this would have disastrous effects on the boundary layer. But this is not so when the body is oscillating periodically. The non-steady behaviour of the pressure field may affect the boundary layer owing to temporal changes in additional local and non-effective oscillations.

\section{Modulation of swimming in frog tadpoles by 5-hydroxytryptamine}

J. Wedderburn, K.T. Sillar \& J. Simmers. Gatty Marine Laboratory, Department of Biology \& Preclinical Medicine, University of St Andrews, St Andrews, Fife, KY16 8LB

In Xenopus frog tadpoles, the first day of larval life witnesses a dramatic change in the motor output underlying swimming. At the time of hatching, the embryo generates a simple swimming rhythm in which motoneurons fire single impulses on each cycle of activity. Although the basic coordination of motor output remains unaltered, the larval rhythm involves bursts of motor discharge on each cycle. In many vertebrates 5-hydroxytryptamine (5HT; serotonin) has been shown to modulate the duration of motor bursts underlying locomotion. The likely source of endogenous transmitter is the raphe nucleus located in the brainstem. We are investigating the possible involvement of $5 \mathrm{HT}$ in rhythm development and modulation.

Using extracellular ventral root recordings we show that the development of ventral root bursts follows a rostrocaudal gradient. Bath applied $5 \mathrm{HT}$ increases burst durations during fictive swimming in larvae but sensitivity to $5 \mathrm{HT}$ also follows a rostrocaudal gradient during development. Since these effects are mimicked by 5 -hydroxytryptophan (5HTP), the metabolic precursor of $5 \mathrm{HT}$, our results suggest that an endogenous $5 \mathrm{HT}$ system located in the raphe nucleus develops rostro-caudally, is preceded by the $5 \mathrm{HT}$ receptor development and may play an important role in the modulation of motor output. 


\section{WORKSHOP}

\section{Fish swimming muscles and movements}

A discussion and workshop convened by J.J. Videler

The Plymouth meeting offers a rare opportunity to discuss the present state of our knowledge on how fish undulatory swimming movements are made. It is a multidisciplinary problem including interesting aspects of the functional morphology of the swimming apparatus, physiological properties of myotome muscle fibres and hydrodynamic interactions between fish and water. Since specialists from all the disciplines involved, from both sides of the Atlantic, are brought together, it is proposed that this opportunity be used to make a collective effort to answer some of the following questions:

(1) What is the function of the complex shape of myotomes and the complex helical configuration of trains of muscle fibres in successive myotomes?

(2) How is the skin, with its layers of crisscross fibres, involved in the transfer of forces to the tail blade?

(3) What is the relation between the timing of the onset and the duration of muscle activity from head to tail and the period of the swimming wave of curvature?

(4) Do fish myotome muscle fibres generate force by shortening, bulging, or both?

(5) Is it true that, during steady swimming, muscle fibres in the caudal peduncle are activated while being stretched (eccentric contraction), the fibres behind the head are contracting isotonically and that the fibres in between contract isometrically?

(6) Are our ideas about the generation of swimming movements coherent with hydrodynamic theories about the interaction between fish and water? 